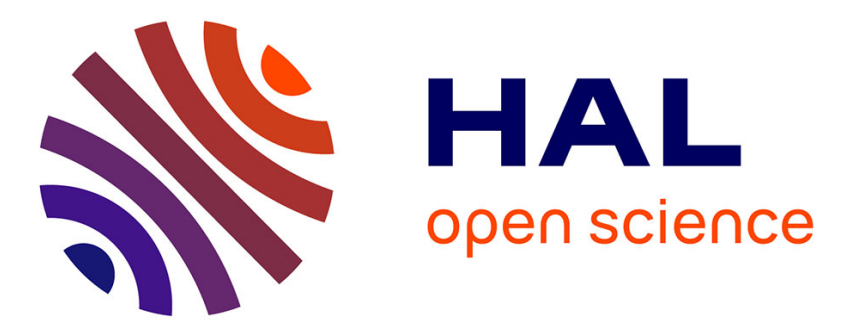

\title{
An all-regime Lagrange-Projection like scheme for 2D homogeneous models for two-phase flows on unstructured meshes
}

Christophe Chalons, Mathieu Girardin, Samuel Kokh

\section{To cite this version:}

Christophe Chalons, Mathieu Girardin, Samuel Kokh. An all-regime Lagrange-Projection like scheme for 2D homogeneous models for two-phase flows on unstructured meshes. Journal of Computational Physics, 2017, 335, pp.885-904. 10.1016/j.jcp.2017.01.017 . hal-01495699

\section{HAL Id: hal-01495699 \\ https://hal.science/hal-01495699}

Submitted on 26 Mar 2017

HAL is a multi-disciplinary open access archive for the deposit and dissemination of scientific research documents, whether they are published or not. The documents may come from teaching and research institutions in France or abroad, or from public or private research centers.
L'archive ouverte pluridisciplinaire HAL, est destinée au dépôt et à la diffusion de documents scientifiques de niveau recherche, publiés ou non, émanant des établissements d'enseignement et de recherche français ou étrangers, des laboratoires publics ou privés. 


\title{
An all-regime Lagrange-Projection like scheme for 2D homogeneous models for two-phase flows on unstructured meshes
}

\author{
Christophe CHALONS ${ }^{\mathrm{a}, *}$, Mathieu GIRARDIN ${ }^{\mathrm{b}, \mathrm{e}}$, Samuel KOKH ${ }^{\mathrm{c}, \mathrm{d}}$ \\ ${ }^{a}$ LMV - UMR 8100, Univ. Versailles Saint-Quentin-en-Yvelines, UFR des Sciences, \\ Bâtiment Fermat, 45 avenue des Etats-Unis, 78035 Versailles cedex, France. \\ ${ }^{b} C E A, D A M, D I F, F-91297$ Arpajon, France \\ ${ }^{c}$ Maison de la Simulation USR 3441, Digiteo Labs, bât 565, PC 190, CEA Saclay, 91191 \\ Gif sur Yvette Cedex, France. \\ ${ }^{d}$ DEN/DANS/DM2S/STMF - CEA Saclay, 91191 Gif sur Yvette Cedex, France. \\ eformerly: DEN/DANS/DM2S/STMF/LMEC CEA Saclay and LRC MANON, Laboratoire \\ de Recherche Conventionné CEA/DEN/DANS/DM2S and UPMC-CNRS/LJLL
}

\begin{abstract}
We propose an all regime Lagrange-Projection like numerical scheme for $2 \mathrm{D}$ homogeneous models for two-phase flows. By all regime, we mean that the numerical scheme is able to compute accurate approximate solutions with an under-resolved discretization, i.e. a mesh size and time step much bigger than the Mach number $M$ of the mixture. The key idea is to decouple acoustic, transport and phase transition phenomenon using a Lagrange-Projection decomposition in order to treat implicitly (fast) acoustic and phase transition phenomenon and explicitly the (slow) transport phenomena. Then, extending a strategy developed in the case of the usual gas dynamics equations, we alter the numerical flux in the acoustic approximation to obtain an uniform truncation error in terms of $M$. This modified scheme is conservative and endowed with good stability properties with respect to the positivity of the density and preserving the mass fraction within the interval $(0,1)$. Numerical evidences are proposed and show the ability of the scheme to deal with cases where the flow regime may vary from low to high Mach values.
\end{abstract}

Keywords: Two-phase flows, low-Mach regime, finite volume schemes, all-regime schemes, Lagrange-Projection like schemes, large time-steps

\footnotetext{
Fully documented templates are available in the elsarticle package on CTAN

* Corresponding author

Email addresses: christophe.chalons@uvsq.fr (Christophe CHALONS), mathieu.girardin2@cea.fr (Mathieu GIRARDIN), samuel.kokh@cea.fr (Samuel KOKH)
} 


\section{Introduction}

The simulation of two-phase flows accounting for mass transfer between a liquid and its vapor phase pertains to a wide range of industrial applications. They may bring to play very different flow regimes stemming from low-velocity steady flows in heat exchangers to water hammer effects in pipes that involve cavitation phenomena near closing valves in pipes. A significant factor for such regime discrepancy lies in the fact that the sound velocity in a two-phase mixture is considerably lower than in the pure phase 11, 2. Therefore, if phase change occurs in a flow it may result in a large variations of the Mach number $M$ across the computational domain.

In the present work, we intend to focus on the simulation of compressible two-phase flows with mass transfer phenomena that may experience important Mach number variations. Among the vast diversity of two-phase flows models, we choose a simple framework that entails phase change by considering a compressible Homogeneous Relaxation Model frequently referred to as HRM and its related Homogeneous Equilibrium Model (HEM), see [3, 4, 5, 6, 7] and references therein. We propose a collocated Finite Volume method that addresses two important issues if one needs to use the same model for both low-velocity and fast dynamics processes.

The first issue concerns the lack of accuracy in the low-Mach regime of Godunov-type schemes when using an under-resolved mesh. This problem has been widely investigated in the case of the gas dynamics equations, see [8], [9], [10, [11 , [12, [13], 14, [15, [16], 17], [18]. The analysis of these authors

may rely on different arguments like the analysis of the viscosity matrix [8], an asymptotic expansion in terms of Mach number [9], a detailed study in [12] that seeks invariance properties of the numerical scheme transposing the framework of Schochet to the discrete setting, and also an analysis based on the so-called Asymptotic Preserving property in [15]. Nevertheless the resulting cure usually boils down to reducing the numerical diffusion in the momentum equation for low Mach number values. Some works have been devoted to the extension of those strategies to two-phase flows [5, 19, 20, 21].

The second problem we address is the CFL restriction on the time step for explicit Finite Volume methods that involve the (fast) acoustic and phase transition phenomena. It seems natural to seek for numerical schemes that enable 
the use of large time steps constrained only by the (slow) material phenomena, see [10, 22, 15, 23, 18].

Numerical schemes that can tackle both issues, namely: accuracy for mesh sizes that do not depend on the Mach number and also stability for time steps that are not constrained by the Mach value are usually referred to as all regime, like the methods proposed in [10, 15, 18].

In the present work, we propose an extension of the method proposed in [18] for the gas dynamics equations to the case of homogeneous models for two-phase flows. An operator splitting strategy allows to decouple the acoustic, transport and phase transition phenomena. The approximation algorithm is split into three steps : an acoustic step, a transport step and a phase transition step. A mixed implicit-explicit method is obtained by using implicit updates for the acoustic and phase transition steps, and an explicit update for the transport step. Then, a modification of fluxes for the acoustic step allow to recover a truncation error that is uniform with respect to the Mach number. The resulting scheme allows to cope with unstructured meshes and compressible flows equipped with very general Equation of State (EOS). Finally, let us mention that the overall procedure is shown to be a conservative discretization (except for the mass fraction due to phase transition) and endowed with good stability properties with respect to the positivity of the density and ensuring that the mass fraction remains in the interval $(0,1)$. We also prove the validity of a discrete entropy inequality in $2 \mathrm{D}$ and for general meshes.

\section{Governing equations and low-Mach number regime}

Governing equations. Let us note $\left(x_{1}, x_{2}\right) \in \mathbb{R}^{2}$ and $\nabla=\left(\partial_{x_{1}} \cdot, \partial_{x_{2}} \cdot\right)$, we are interested in the two-dimensional homogeneous relaxation model (HRM)

$$
\left\{\begin{aligned}
\partial_{t}(\rho Y)+\nabla \cdot(\rho Y \mathbf{u}) & =\lambda_{0} \rho\left(Y^{*}(\rho, e)-Y\right) \\
\partial_{t} \rho+\nabla \cdot(\rho \mathbf{u}) & =0 \\
\partial_{t}(\rho \mathbf{u})+\nabla \cdot(\rho \mathbf{u} \otimes \mathbf{u})+\nabla p & =0 \\
\partial_{t}(\rho E)+\nabla \cdot[(\rho E+p) \mathbf{u}] & =0
\end{aligned}\right.
$$

where $Y, \rho, \mathbf{u}=(u, v)^{t}, E$ denotes the mass fraction, the density, the velocity vector and the total energy of the mixture. The pressure $p=p^{\mathrm{HRM}}(\rho, e, Y)$ is assumed to be a given function of the density $\rho$, the internal energy $e=E-\frac{|\mathbf{u}|^{2}}{2}$ of the mixture and the mass fraction $Y$. The mass fraction at thermodynamic 
equilibrium $Y^{*}(\rho, e)$ is a given function of the density and the internal energy of the mixture. For HRM, the thermodynamic equilibrium $Y=Y^{*}(\rho, e)$ is not instantaneously achieved but is reached at relaxation rate $\lambda_{0}>0$. We refer for instance the reader to [3, 4, 5, 6] and the references therein. We make the assumption that $c^{2}=\partial_{\rho} p^{\mathrm{HRM}}+p^{\mathrm{HRM}} \partial_{e} p^{\mathrm{HRM}} / \rho^{2}>0$, where $c$ is the sound velocity of the (HRM) system.

Remark 1. We note that in the limit $\lambda_{0} \rightarrow \infty$, the (HRM) system (1) converges at least formally toward the homogeneous equilibrium model (HEM) given by

$$
\left\{\begin{aligned}
Y & =Y^{*}(\rho, e), \\
\partial_{t} \rho+\nabla \cdot(\rho \mathbf{u}) & =0 \\
\partial_{t}(\rho \mathbf{u})+\nabla \cdot(\rho \mathbf{u} \otimes \mathbf{u})+\nabla p^{H E M} & =0 \\
\partial_{t}(\rho E)+\nabla \cdot\left[\left(\rho E+p^{H E M}\right) \mathbf{u}\right] & =0
\end{aligned}\right.
$$

where $p^{H E M}(\rho, e)=p^{H R M}\left(\rho, e, Y^{*}(\rho, e)\right)$.

Dimensionless governing equations. We are now interested in the behavior of the HRM system with respect to the variation of the Mach regime. In order to characterize the Mach regime of the flow, we consider a rescaling of the equations. Let us introduce the following non-dimensional quantities :

$\tilde{x}_{1}=\frac{x_{1}}{L}, \quad \tilde{x}_{2}=\frac{x_{2}}{L}, \quad \tilde{t}=\frac{t}{T}, \quad \tilde{\rho}=\frac{\rho}{\rho_{0}}, \quad \tilde{u}=\frac{u}{u_{0}}, \quad \tilde{v}=\frac{v}{v_{0}}, \quad \tilde{e}=\frac{e}{e_{0}}, \quad \tilde{p}=\frac{p}{p_{0}}, \quad \tilde{c}=\frac{c}{c_{0}}$.

The parameters $L, T, u_{0}=v_{0}=\frac{L}{T}, \rho_{0}, e_{0}=\rho_{0} p_{0}$ and $c_{0}=\sqrt{\frac{p_{0}}{\rho_{0}}}$ denote respectively a characteristic length, time, velocity, density, internal energy, pressure, and sound speed of the mixture. If $M=\frac{u_{0}}{c_{0}}$ is the so-called Mach-number and if we note $\tilde{\nabla}=\left(\partial_{\tilde{x}_{1}} \cdot, \partial_{\tilde{x}_{2}} \cdot\right)$, then system [1] reads

$$
\left\{\begin{aligned}
\partial_{\tilde{t}}(\tilde{\rho} Y)+\tilde{\nabla} \cdot(\tilde{\rho} Y \tilde{\mathbf{u}})-\lambda_{0} T \tilde{\rho}\left(Y^{*}\left(\rho_{0} \tilde{\rho}, e_{0} \tilde{e}\right)-Y\right) & =0, \\
\partial_{\tilde{t}} \tilde{\rho}+\tilde{\nabla} \cdot(\tilde{\rho} \tilde{\mathbf{u}}) & =0, \\
\partial_{\tilde{t}}(\tilde{\rho} \tilde{\mathbf{u}})+\tilde{\nabla} \cdot(\tilde{\rho} \tilde{\mathbf{u}} \otimes \tilde{\mathbf{u}})+\frac{1}{M^{2}} \tilde{\nabla} \tilde{p} & =0 \\
\partial_{\tilde{t}}(\tilde{\rho} \tilde{e})+\tilde{\nabla} \cdot[(\tilde{\rho} \tilde{e}+\tilde{p}) \tilde{\mathbf{u}}]+\frac{M^{2}}{2}\left[\partial_{\tilde{t}}\left(\tilde{\rho}|\tilde{\mathbf{u}}|^{2}\right)+\tilde{\nabla} \cdot\left(\tilde{\rho}|\tilde{\mathbf{u}}|^{2} \tilde{\mathbf{u}}\right)\right] & =0,
\end{aligned}\right.
$$

This system motivates the following definition.

Definition 1. In the following, the flow is said to be in the low-Mach regime if and only if the Mach number $M \ll 1$ and $\tilde{\nabla} \tilde{p}=\mathcal{O}\left(M^{2}\right)$. 
Remark 2. If the term $\tilde{\nabla} \tilde{p}$ does not remain of magnitude $\mathcal{O}\left(M^{2}\right)$ then the variation of $\tilde{\rho} \tilde{u}$ will reach a magnitude $\mathcal{O}\left(\frac{1}{M}\right)$ or $\mathcal{O}\left(\frac{1}{M^{2}}\right)$. These large magnitude variations of the momentum will induce a growth of the Mach number and thus change the Mach regime.

Remark 3. The source term in the mass fraction equation may be stiff if the relaxation toward thermodynamic equilibrium is much faster than the convective part of the system $\left(\lambda_{0} T \gg 1\right)$.

Remark 4. Let us underline that the definition of a relevant Mach number, and a fortiori the definition of a low Mach regime for two-phase flow models is not a trivial matter in the general case. Indeed, many models involve several material velocities and several sound velocities, like in the Baer-Nunziato model [24] or the model of Cheng, Drew and Lahey [25]. Nevertheless, in the case of the HEM or HRM models without velocity drift law, there is a natural definition for the sound velocity, the Mach number and the low Mach regime.

Before going any further, let us underline that the present work does not involve the study of the rescaled system (3) in the limit regime $M \rightarrow 0$. This delicate question has been widely investigated over the past years and is still a rich field of research [9, 15, 19]. We focus here on a simpler task that consists in examining the consistency with (3) of a rescaled numerical scheme in the low Mach regime. We shall see that this only involves evaluating the truncation error of the rescaled numerical scheme (in the sense of Finite Difference) and determining how it depends on the Mach number. Let us emphasize that this procedure only relies on a local behavior of the solution and does not require specific hypotheses besides the (local) smoothness of the solution and the (local) low Mach regime hypothesis.

\section{Acoustic-transport-phase transition operator splitting strategy}

In this section, we propose a three-step approximation strategy based on an operator splitting for approximating the solutions of (1). The aim of this splitting is to decouple acoustic, transport and phase transition phenomena. Using the chain rule for the space derivatives, we split system (1) into the following three subsystems. The first subsystem describe the transport process 
and reads

$$
\left\{\begin{array}{l}
\partial_{t}(\rho Y)+\mathbf{u} \cdot \nabla(\rho Y)=0 \\
\partial_{t} \rho+\mathbf{u} \cdot \nabla(\rho)=0 \\
\partial_{t}(\rho \mathbf{u})+\mathbf{u} \cdot \nabla(\rho \mathbf{u})=0 \\
\partial_{t}(\rho E)+\mathbf{u} \cdot \nabla(\rho E)=0
\end{array}\right.
$$

The second subsystem governs the acoustic phenomena, namely

$$
\begin{aligned}
\partial_{t}(\rho Y)+(\rho Y) \nabla \cdot \mathbf{u} & =0, & \partial_{t} \rho+\rho \nabla \cdot \mathbf{u} & =0, \\
\partial_{t}(\rho \mathbf{u})+(\rho \mathbf{u}) \nabla \cdot \mathbf{u}+\nabla p & =0, & \partial_{t}(\rho E)+(\rho E) \nabla \cdot \mathbf{u}+\nabla \cdot[p \mathbf{u}] & =0,
\end{aligned}
$$

Or equivalently with $\tau=\frac{1}{\rho}$ the specific volume

$$
\left\{\begin{aligned}
\partial_{t} Y & =0 \\
\partial_{t} \tau-\tau \nabla \cdot \mathbf{u} & =0 \\
\partial_{t} \mathbf{u}+\tau \nabla p & =0 \\
\partial_{t} E+\tau \nabla \cdot(p \mathbf{u}) & =0
\end{aligned}\right.
$$

This system is nothing but the gas dynamics equations in Lagrangian coordinates, so that the proposed transport-acoustic decomposition is nothing but the natural (and physically relevant) Lagrange-Projection strategy. This is an original approach for treating low Mach regimes that was first proposed in [18, other works using a fixed-mesh Lagrange-Projection splitting have also been presented in [26, 27].

The third subsystem accounts for mass transfer between phases and reads

$$
\left\{\begin{aligned}
\partial_{t}(\rho Y) & =\lambda_{0} \rho\left(Y^{*}(\rho, e)-Y\right) \\
\partial_{t} \rho & =0 \\
\partial_{t}(\rho \mathbf{u}) & =0 \\
\partial_{t}(\rho E) & =0
\end{aligned}\right.
$$

or equivalently

$$
\left\{\begin{aligned}
\partial_{t} Y & =\lambda_{0}\left(Y^{*}(\rho, e)-Y\right), \\
\partial_{t} \rho & =0 \\
\partial_{t}(\rho \mathbf{u}) & =0 \\
\partial_{t}(\rho E) & =0
\end{aligned}\right.
$$

Let us mention that this transport/acoustic/phase transition splitting separates physical phenomena that happen at speed $u_{0} / c_{0} / \lambda_{0}$ that may differ from several order of magnitude. From a numerical point of view, such a decomposition 
is very helpful to design large time step implicit-explicit strategy with CFL restriction based only on the slow phenomenon [22, 23, 18].

\section{Numerical scheme}

Let us suppose that the domain $\Omega \subset \mathbb{R}^{2}$ is discretized by $N$ cells $\Omega_{i}$. Let $\Gamma_{i j}$ be the common edge of two neighbouring cells $\Omega_{i}$ and $\Omega_{j}$ and $\mathbf{n}_{i j}$ be the unit vector normal to $\Gamma_{i j}$ pointing from $\Omega_{i}$ to $\Omega_{j}$. We define $N(i)$ the set of indices $1 \leq j \leq N$ such that $\Omega_{i}$ and $\Omega_{j}$ have a common face (figure 4 ). Let $\Delta t>0$ be the time step, we define the intermediate times $t^{n}=n \Delta t$ for $n \in \mathbb{N}$. If $b$ is a fluid parameter, in the sequel, we will note $b_{i}^{n}\left(\right.$ resp. $\left.b_{i}^{n+1}\right)$ the approximate value of $b$ within the cell $\Omega_{i}$ at instant $t=t^{n}$ (resp. $\left.t=t^{n+1}\right)$.

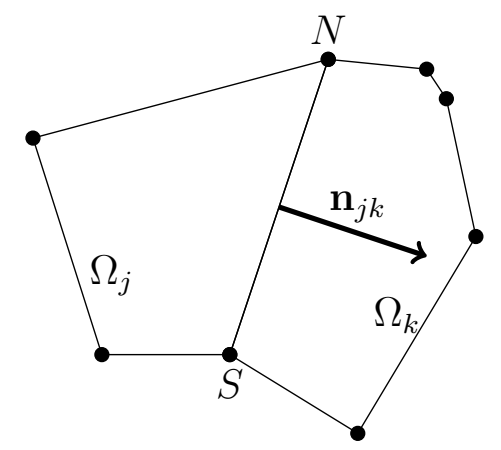

Figure 1: the face $\Gamma_{j k}=\overline{\Omega_{j}} \cap \overline{\Omega_{k}}$ defined the segment $(N S)$ has a unit normal vector $\mathbf{n}_{j k}$ oriented from $\Omega_{j}$ to $\Omega_{k}$.

Given a fluid state $(Y, \rho, \rho u, \rho v, \rho E)_{i}^{n}, 1 \leq i \leq N$ at instant $t^{n}$, this splitting algorithm may be decomposed as follows

1. Acoustic step : Update the fluid state $(Y, \rho, \rho u, \rho v, \rho E)_{i}^{n}$ to the value $(Y, \rho, \rho u, \rho v, \rho E)_{i}^{n+1-}$ by approximating the solution of (5);

2. Transport step : Update the fluid state $(Y, \rho, \rho u, \rho v, \rho E)_{i}^{n+1-}$ to the value $(\bar{Y}, \bar{\rho}, \overline{\rho u}, \overline{\rho v}, \overline{\rho E})_{i}$ by approximating the solution of $(4)$;

3. Phase transition step : Update the fluid state $(\bar{Y}, \bar{\rho}, \overline{\rho u}, \overline{\rho v}, \overline{\rho E})_{i}$ to the value $(Y, \rho, \rho u, \rho v, \rho E)_{i}^{n+1}$ by approximating the solution of $(6)$.

Remark 5. It is interesting to note that a different order of splitting could have been considered a priori in order to approximate the three operators. However, the proposed order enjoys the following desirable properties: first, it yields a 
conservative discretization for the global mass, the momentum and the total energy. This feature is important for simulations of flows developing shocks in the computational domain. Moreover, in the limit $\lambda_{0} \rightarrow+\infty$, the third step that accounts for mass transfer boils down to restoring the $Y=Y^{*}$ equilibrium for the HEM system, before evaluating the pressure and the sound velocity of the Lagrange step. These nice properties are not natural with a different order of splitting.

Let us now detail each step of the algorithm.

Acoustic step (Lagrange step). Regarding the acoustic step (5) we propose the following update formulas

$$
\left\{\begin{array}{l}
\mathbf{u}_{i}^{n+1-}=\mathbf{u}_{i}^{n}-\tau_{i}^{n} \Delta t \sum_{j \in N(i)} \sigma_{i j} p_{i j}^{*, \theta} \mathbf{n}_{i j} \\
\Pi_{i}^{n+1-}=\Pi_{i}^{n}-\tau_{i}^{n} \Delta t \sum_{j \in N(i)} \sigma_{i j}\left(a_{i j}\right)^{2} u_{i j}^{*} \\
Y_{i}^{n+1-}=Y_{i}^{n}, \\
\tau_{i}^{n+1-}=\tau_{i}^{n}+\tau_{i}^{n} \Delta t \sum_{j \in N(i)} \sigma_{i j} u_{i j}^{*} \\
E_{i}^{n+1-}=E_{i}^{n}-\tau_{i}^{n} \Delta t \sum_{j \in N(i)} \sigma_{i j} p_{i j}^{*, \theta} u_{i j}^{*}
\end{array}\right.
$$

where $\sigma_{i j}=\left|\Gamma_{i j}\right| /\left|\Omega_{i}\right|$ and $\Pi$ is an unknown associated with the so-called Suliciu relaxation approximation and given at time $t^{n}$ by $\Pi_{i}^{n}=p\left(\rho_{i}^{n}, e_{i}^{n}, Y_{i}^{n}\right)$, see [28, 29, 30.

The three scalar quantities $a_{i j}, p_{i j}^{*, \theta}$, and $u_{i j}^{*}$ represent respectively an average sound velocity, a pressure and normal velocity at the face $\Gamma_{i j}$ and are given by

$$
\left\{\begin{array}{l}
a_{i j}=\max \left((\rho c)_{i}^{n},(\rho c)_{j}^{n}\right), \\
u_{i j}^{*}=\frac{1}{2} \mathbf{n}_{i j}^{T}\left(\mathbf{u}_{i}^{n}+\mathbf{u}_{j}^{n}\right)-\frac{1}{2 a_{i j}}\left(\Pi_{j}^{n}-\Pi_{i}^{n}\right), \\
p_{i j}^{*, \theta}=\frac{1}{2}\left(\Pi_{i}^{n}+\Pi_{j}^{n}\right)-\frac{a_{i j} \theta_{i j}}{2} \mathbf{n}_{i j}^{T}\left(\mathbf{u}_{j}^{n}-\mathbf{u}_{i}^{n}\right) .
\end{array}\right.
$$

We remark that the modification of classical fluxes thanks to $\theta_{i j}$ will allow to avoid spurious numerical diffusion in the low Mach regime, as proposed recently in [18] such a modification is the key point to make the scheme accurate in the low Mach regime. The classical Suliciu relaxation fluxes correspond to the choice $\theta_{i j}=1$.

At this stage, the CFL restriction of this explicit scheme is based on the (fast) 
acoustic waves and reads

$$
\Delta t \max _{1 \leq j \leq N}\left[\tau_{j}^{n}\left(\max _{i \in N(j)} \sigma_{i j} a_{i j}\right)\right] \leq \frac{1}{2}
$$

To obtain a time step definition based only on the slow waves, following ideas developed in [22, 23, 18, we propose to use an implicit scheme for the acoustic step. We use (7) with a new definition of the pressure and normal velocity at the interface $\Gamma_{i j}$ given by

$$
\left\{\begin{aligned}
a_{i j} & =\max \left((\rho c)_{i}^{n},(\rho c)_{j}^{n}\right) \\
u_{i j}^{*} & =\frac{1}{2} \mathbf{n}_{i j}^{T}\left(\mathbf{u}_{i}^{n+1-}+\mathbf{u}_{j}^{n+1-}\right)-\frac{1}{2 a_{i j}}\left(\Pi_{j}^{n+1-}-\Pi_{i}^{n+1-}\right), \\
p_{i j}^{*, \theta} & =\frac{1}{2}\left(\Pi_{i}^{n+1-}+\Pi_{j}^{n+1-}\right)-\frac{a_{i j} \theta_{i j}}{2} \mathbf{n}_{i j}^{T}\left(\mathbf{u}_{j}^{n+1-}-\mathbf{u}_{i}^{n+1-}\right) .
\end{aligned}\right.
$$

Thanks to the Suliciu-type relaxation strategy, scheme $(7)-(10)$ is valid for any pressure law and only requires to solve a linear problem with respect to variables $\mathbf{u}$ and $\Pi$. Then other update formulas for variables $Y, \tau$ and $E$ are evaluated explicitly, while the scheme is actually implicit.

Transport step (Projection step). In order to approximate the solutions of (4), we simply use an upwind Finite-Volume scheme : Let $\varphi \in\{\rho Y, \rho, \rho \mathbf{u}, \rho E\}$, we set

$$
\bar{\varphi}_{i}=\varphi_{i}^{n+1-}-\Delta t\left[\sum_{j \in N(i)}\left(\sigma_{i j} u_{i j}^{*} \varphi_{i j}^{n+1-}\right)\right]+\Delta t \varphi_{i}^{n+1-}\left[\sum_{j \in N(i)}\left(\sigma_{i j} u_{i j}^{*}\right)\right],
$$

where $\varphi_{i j}^{n+1-}$ is defined by the upwind choice with respect to the sign of $u_{i j}^{*}$, namely

$$
\varphi_{i j}^{n+1-}= \begin{cases}\varphi_{i}^{n+1-}, & \text { if } u_{i j}^{*}>0 \\ \varphi_{j}^{n+1-}, & \text { if } u_{i j}^{*} \leq 0 .\end{cases}
$$

The CFL restriction of this explicit scheme is based on the (slow) material waves and reads

$$
\Delta t \max _{1 \leq j \leq N}\left[\sum_{i \in N(j)}\left(\sigma_{i j}\left|u_{i j}^{*}\right|\right)\right] \leq 1
$$

Phase transition step (Source terms step). To approximate system (6), 
we propose a pointwise implicit evaluation

$$
\left\{\begin{array}{l}
Y_{i}^{n+1}=\bar{Y}_{i}+\lambda_{0} \Delta t\left(Y^{*}\left(\bar{\rho}_{i}, \bar{e}_{i}\right)-Y_{i}^{n+1}\right) \\
\varphi_{i}^{n+1}=\bar{\varphi}_{i}, \quad \varphi \in\{\rho, \rho \mathbf{u}, \rho E\}
\end{array}\right.
$$

The implicit treatment is particularly important for large values of $\lambda_{0}$ to avoid a CFL restriction based on the (fast) phase transition phenomenon.

Remark 6. In the limit $\lambda_{0} \rightarrow+\infty$, this step may be replaced by the projection on the thermodynamic equilibrium

$$
\left\{\begin{array}{l}
Y_{i}^{n+1}=Y^{*}\left(\bar{\rho}_{i}, \bar{e}_{i}\right) \\
\varphi_{i}^{n+1}=\bar{\varphi}_{i}, \quad \varphi \in\{\rho, \rho \mathbf{u}, \rho E\}
\end{array}\right.
$$

to obtain a numerical scheme for the HEM system (2).

Overall numerical scheme. The overall numerical scheme composed by the discretization $(7)-(11)-(14)$ is conservative with respect to the variables $\rho$, $\rho \mathbf{u}, \rho E$ for both the implicit solver $(10)$ and the explicit solver (8). The update from $t^{n}$ to $t^{n+1}$ reads after easy calculations

$$
\left\{\begin{array}{l}
(\rho Y)_{i}^{n+1}=(\rho Y)_{i}^{n}-\Delta t \sum_{j \in N(i)} \sigma_{i j}(\rho Y)_{i j}^{n+1-} u_{i j}^{*}+\lambda_{0} \Delta t \rho_{i}^{n+1}\left(Y^{*}\left(\bar{\rho}_{i}, \bar{e}_{i}\right)-Y_{i}^{n+1}\right) \\
\rho_{i}^{n+1}=\rho_{i}^{n} \quad-\Delta t \sum_{j \in N(i)} \sigma_{i j} \rho_{i j}^{n+1-} u_{i j}^{*} \\
(\rho \mathbf{u})_{i}^{n+1}=(\rho \mathbf{u})_{i}^{n}-\Delta t \sum_{j \in N(i)} \sigma_{i j}\left((\rho \mathbf{u})_{i j}^{n+1-} u_{i j}^{*}+p_{i j}^{*, \theta} \mathbf{n}_{i j}\right) \\
(\rho E)_{i}^{n+1}=(\rho E)_{i}^{n}-\Delta t \sum_{j \in N(i)} \sigma_{i j}\left((\rho E)_{i}^{n+1-}+p_{i j}^{*, \theta}\right) u_{i j}^{*}
\end{array}\right.
$$

For the sake of clarity, let us briefly recall the different steps of the method that shall be referred to as $\operatorname{LPS}-\operatorname{IMEX}(\theta)$. Assume that $(\rho Y, \rho, \rho \mathbf{u}, \rho E)_{j}^{n}$ is known, $(\rho Y, \rho, \rho \mathbf{u}, \rho E)_{j}^{n+1}$ is computed by the following three steps :

(i) compute $(\rho Y, \rho, \rho \mathbf{u}, \rho E)_{j}^{n+1-}$ from $(\rho Y, \rho, \rho \mathbf{u}, \rho E)_{j}^{n}$ with $(7)-(10)$,

(ii) compute $(\overline{\rho Y}, \bar{\rho}, \overline{\rho \mathbf{u}}, \overline{\rho E})_{j}$ from $(\rho Y, \rho, \rho \mathbf{u}, \rho E)_{j}^{n+1-}$ with $(11)-(12)$,

(iii) compute $(\rho Y, \rho, \rho \mathbf{u}, \rho E)_{j}^{n+1}$ from $(\overline{\rho Y}, \bar{\rho}, \overline{\rho \mathbf{u}}, \overline{\rho E})_{j}$ with 14 for HRM or with 15 for HEM.

We also define the method that shall be referred to as LPS-EX $(\theta)$. Assume that $(\rho Y, \rho, \rho \mathbf{u}, \rho E)_{j}^{n}$ is known, $(\rho Y, \rho, \rho \mathbf{u}, \rho E)_{j}^{n+1}$ is computed by the following three steps : 
(i) compute $(\rho Y, \rho, \rho \mathbf{u}, \rho E)_{j}^{n+1-}$ from $(\rho Y, \rho, \rho \mathbf{u}, \rho E)_{j}^{n}$ with $(7)-(8)$,

(ii) compute $(\overline{\rho Y}, \bar{\rho}, \overline{\rho \mathbf{u}}, \overline{\rho E})_{j}$ from $(\rho Y, \rho, \rho \mathbf{u}, \rho E)_{j}^{n+1-}$ with $\left.\overline{11}\right)-\sqrt{12}$,

(iii) compute $(\rho Y, \rho, \rho \mathbf{u}, \rho E)_{j}^{n+1}$ from $(\overline{\rho Y}, \bar{\rho}, \overline{\rho \mathbf{u}}, \overline{\rho E})_{j}$ with 14 for HRM or with $(15)$ for HEM.

The difference between these methods is that the Lagrange step is implicit for the $\operatorname{LPS}-\operatorname{IMEX}(\theta)$ scheme and explicit for the $\operatorname{LPS}-\operatorname{EX}(\theta)$ scheme. The source terms step is treated implicitly and the transport step explicitly in both schemes.

\section{Main properties}

We now give the main properties of the $\operatorname{LPS}-\operatorname{EX}(\theta)$ and $\operatorname{LPS}-\operatorname{IMEX}(\theta)$ schemes.

Theorem 2. Under the acoustic CFL condition (9) and the material CFL condition (13), the LPS-EX( $\theta)$ scheme is well-defined and satisfies the following properties

(i) it is a conservative scheme for $\rho, \rho \mathbf{u}$ and $\rho E$. It is also a conservative scheme for $\rho Y$ when there is no mass transfer between phases $\left(\lambda_{0}=0\right)$.

(ii) the density $\rho_{i}^{n}$ is positive for all $i$ and $n>0$ provided that $\rho_{i}^{0}$ is positive for all $i$.

(iii) $Y_{i}^{n} \in[0,1]$ for all $i$ and $n>0$ provided that $Y_{i}^{0} \in[0,1]$ for all $i$ and $\bar{e}_{i}>0$ for all $i$ and $n \geq 0$.

(iv) if $\theta=\mathcal{O}(M)$, then the truncation error of the numerical scheme is uniform with respect to $M<1$.

(v) it is not stable in the uniform sense with respect to the Mach number $M$.

Let us underline that the acoustic CFL condition (9) is independent of the modification $\theta$.

Theorem 3. Under the material CFL condition (13), the LPS-IMEX( $\theta)$ scheme is well-defined and satisfy the following properties

(i) it is a conservative scheme for $\rho, \rho \mathbf{u}$ and $\rho E$. It is also a conservative scheme for $\rho Y$ when there is no mass transfer between phases $\left(\lambda_{0}=0\right)$.

(ii) the density $\rho_{i}^{n}$ is positive for all $i$ and $n>0$ provided that $\rho_{i}^{0}$ is positive for all $i$.

(iii) $Y_{i}^{n} \in[0,1]$ for all $i$ and $n>0$ provided that $Y_{i}^{0} \in[0,1]$ for all $i$ and $\bar{e}_{i}>0$ for all $i$ and $n \geq 0$. 
(iv) if $\theta=\mathcal{O}(M)$, then the truncation error of the numerical scheme is uniform with respect to $M<1$.

(v) it is stable in the uniform sense with respect to the Mach number $M$.

(vi) the linear system with respect to the variables $\mathbf{u}_{i}^{n+1-}$ and $\Pi_{i}^{n+1-}$ issued from equations (7) and (10) and supplemented by Neumann boundary conditions admits a unique solution for any choice of $\Delta t \geq 0$ and $\theta \geq 0$.

Remark 7. For the LPS-EX( $\theta)$ scheme, we may prove the positivity of the internal energy and a discrete entropy inequality under a condition on the modification $\theta$, see appendix Appendix B for more details. Under this condition, we have in particular $\bar{e}_{i}>0$ for all $i$ and $n \geq 0$.

Proof of property (i) is easily obtained from 116 and is thus left to the reader (see also [18]). Proof of property (vi) may be found in [18].

Proof of properties (ii) and (iii). Let us consider that $Y_{i}^{n} \in[0,1], \rho_{i}^{n}>0$ and $\bar{e}_{i}>0$ for all $i$, we are going to show that $Y_{i}^{n+1} \in[0,1]$ and $\rho_{i}^{n+1}>0$ for all $i$ :

- Acoustic step : the mass fraction is unchanged in this step $Y_{i}^{n+1-}=Y_{i}^{n}$. Thus, we have $Y_{i}^{n+1-} \in[0,1]$ for all $i$.

The density is given by

$$
\rho_{i}^{n+1-}=\rho_{i}^{n}\left(1+\Delta t \sum_{j \in N(i)} \sigma_{i j} u_{i j}^{*}\right)^{-1},
$$

so that we have $\rho_{i}^{n+1-}>0$ for all $i$ thanks to the CFL condition 13 .

- Transport step : the upwind choice 12 is such that

$$
u_{i j}^{*} \varphi_{i j}^{n+1-}=\left(u_{i j}^{*}\right)^{+} \varphi_{i}^{n+1-}+\left(u_{i j}^{*}\right)^{-} \varphi_{j}^{n+1-},
$$

where $u^{+}=\frac{u+|u|}{2}$ and $u^{-}=\frac{u-|u|}{2}$. Injecting those expressions in the transport step 11) for the density and the mass fraction holds

$$
\left\{\begin{array}{l}
\bar{\rho}_{i}=\left(1+\Delta t \sum_{j \in N(i)} \sigma_{i j}\left(u_{i j}^{*}\right)^{-}\right) \rho_{i}^{n+1-}-\Delta t \sum_{j \in N(i)} \sigma_{i j}\left(u_{i j}^{*}\right)^{-} \rho_{j}^{n+1-}, \\
\bar{Y}_{i}=\left(\frac{\rho_{i}^{n+1-}}{\bar{\rho}_{i}}\right)\left(1+\Delta t \sum_{j \in N(i)} \sigma_{i j}\left(u_{i j}^{*}\right)^{-}\right) Y_{i}^{n+1-}-\Delta t \sum_{j \in N(i)} \sigma_{i j}\left(u_{i j}^{*}\right)^{-}\left(\frac{\rho_{j}^{n+1-}}{\bar{\rho}_{i}}\right) Y_{j}^{n+1-} .
\end{array}\right.
$$


As $\left(u_{i j}^{*}\right)^{-} \leq 0$, under the CFL condition 13$), \bar{\rho}_{i}$ (resp. $\bar{Y}_{i}$ ) is a convex combination of $\rho_{i}^{n+1-}\left(\operatorname{resp} . Y_{i}^{n+1-}\right)$ and $\rho_{j}^{n+1-}\left(\operatorname{resp} . Y_{j}^{n+1-}\right)$ for $j \in$ $N(i)$. Thus, we have $\bar{\rho}_{i}>0$ and $\bar{Y}_{i} \in[0,1]$ for all $i$.

- Phase transition step : the density is unchanged in this step $\rho_{i}^{n+1}=\bar{\rho}_{i}$, so that $\rho_{i}^{n+1}>0$ for all $i$.

The mass fraction update writes for HRM

$$
Y_{i}^{n+1}=\left(\frac{1}{1+\lambda_{0} \Delta t}\right) \bar{Y}_{i}+\left(\frac{\lambda_{0} \Delta t}{1+\lambda_{0} \Delta t}\right) Y^{*}\left(\bar{\rho}_{i}, \bar{e}_{i}\right)
$$

and for HEM

$$
Y_{i}^{n+1}=Y^{*}\left(\bar{\rho}_{i}, \bar{e}_{i}\right),
$$

In both cases, $Y_{i}^{n+1}$ may be seen as a convex combination of $Y^{*}\left(\bar{\rho}_{i}, \bar{e}_{i}\right)$ and $\bar{Y}_{i}$. We assumed that $\bar{e}_{i}>0$ and proved that $\bar{\rho}_{i}>0$ for all $i$, so that $Y^{*}\left(\bar{\rho}_{i}, \bar{e}_{i}\right) \in[0,1]$ by definition of the function $Y^{*}$. Thus $Y_{i}^{n+1} \in[0,1]$ for all $i$.

This conclude the proof.

Behavior with respect to the Mach regime. In order to prove (iv) and (v), we are now interested in the behavior of the numerical scheme with respect to the Mach regime. Namely, we study the dependence with respect to the Mach number $M$ of both the CFL stability condition and the truncation error.

Introducing the rescaling and tilde variables defined earlier into (8) we get

$$
\left\{\begin{array}{l}
\tilde{u}_{i j}^{*}=\frac{1}{2} \mathbf{n}_{i j}^{T}\left(\tilde{\mathbf{u}}_{i}^{n}+\tilde{\mathbf{u}}_{j}^{n}\right)-\frac{1}{M} \frac{1}{2 \tilde{a}_{i j}}\left(\tilde{\Pi}_{j}^{n}-\tilde{\Pi}_{i}^{n}\right), \\
\tilde{p}_{i j}^{*, \theta}=\frac{1}{2}\left(\tilde{\Pi}_{i}^{n}+\tilde{\Pi}_{j}^{n}\right)-M \frac{\tilde{a}_{i j} \theta_{i j}}{2} \mathbf{n}_{i j}^{T}\left(\tilde{\mathbf{u}}_{j}^{n}-\tilde{\mathbf{u}}_{i}^{n}\right) .
\end{array}\right.
$$

For 10 we get

$$
\left\{\begin{array}{l}
\tilde{u}_{i j}^{*}=\frac{1}{2} \mathbf{n}_{i j}^{T}\left(\tilde{\mathbf{u}}_{i}^{n+1-}+\tilde{\mathbf{u}}_{j}^{n+1-}\right)-\frac{1}{M} \frac{1}{2 \tilde{a}_{i j}}\left(\tilde{\Pi}_{j}^{n+1-}-\tilde{\Pi}_{i}^{n+1-}\right), \\
\tilde{p}_{i j}^{*, \theta}=\frac{1}{2}\left(\tilde{\Pi}_{i}^{n+1-}+\tilde{\Pi}_{j}^{n+1-}\right)-M \frac{\tilde{a}_{i j} \theta_{i j}}{2} \mathbf{n}_{i j}^{T}\left(\tilde{\mathbf{u}}_{j}^{n+1-}-\tilde{\mathbf{u}}_{i}^{n+1-}\right) .
\end{array}\right.
$$

The rescaling of the acoustic step (7) reads 


$$
\left\{\begin{array}{l}
\tilde{\mathbf{u}}_{i}^{n+1-}=\tilde{\mathbf{u}}_{i}^{n}-\frac{1}{M^{2}} \tilde{\tau}_{i}^{n} \Delta \tilde{t} \sum_{j \in N(i)} \tilde{\sigma}_{i j} \tilde{p}_{i j}^{*, \theta} \mathbf{n}_{i j}, \\
\tilde{\Pi}_{i}^{n+1-}=\tilde{\Pi}_{i}^{n}-\tilde{\tau}_{i}^{n} \Delta \tilde{t} \sum_{j \in N(i)} \tilde{\sigma}_{i j}\left(\tilde{a}_{i j}\right)^{2} \tilde{u}_{i j}^{*} \\
Y_{i}^{n+1-}=Y_{i}^{n}, \\
\tilde{\tau}_{i}^{n+1-}=\tilde{\tau}_{i}^{n}+\tilde{\tau}_{i}^{n} \Delta \tilde{t} \sum_{j \in N(i)} \tilde{\sigma}_{i j} \tilde{u}_{i j}^{*}, \\
\tilde{E}_{i}^{n+1-}=\tilde{E}_{i}^{n}-\tilde{\tau}_{i}^{n} \Delta \tilde{t} \sum_{j \in N(i)} \tilde{\sigma}_{i j} \tilde{p}_{i j}^{*, \theta} \tilde{u}_{i j}^{*},
\end{array}\right.
$$

where $\tilde{\sigma_{i j}}=\frac{\sigma_{i j}}{L}$ and $\tilde{a}_{i j}=\frac{a_{i j}}{\rho_{0} c_{0}}$. Note that the CFL restriction of the explicit acoustic step reads now

$$
\Delta \tilde{t} \max _{1 \leq j \leq N}\left[\tilde{\tau}_{j}^{n}\left(\max _{i \in N(j)} \tilde{\sigma}_{i j} \tilde{a}_{i j}\right)\right] \leq \frac{M}{2} .
$$

The rescaling of the transport step 11 reads for $\tilde{\varphi} \in\{\tilde{\rho} Y, \tilde{\rho}, \tilde{\rho} \tilde{\mathbf{u}}, \tilde{\rho} \tilde{E}\}$

$$
\overline{\tilde{\varphi}}_{i}=\tilde{\varphi}_{i}^{n+1-}-\Delta \tilde{t}\left[\sum_{j \in N(i)} \tilde{\sigma_{i j}} \tilde{u}_{i j}^{*} \tilde{\varphi}_{i j}^{n+1-}\right]+\Delta \tilde{t} \tilde{\varphi}_{i}^{n+1-}\left[\sum_{j \in N(i)} \tilde{\sigma_{i j}} \tilde{u}_{i j}^{*}\right] .
$$

The CFL restriction associated with the transport step is

$$
\Delta \tilde{t} \max _{1 \leq j \leq N}\left[\sum_{i \in N(i)}\left(\tilde{\sigma_{i j}}\left|\tilde{u}_{i j}^{*}\right|\right)\right] \leq 1
$$

Finally the phase transition step 14 becomes

$$
\left\{\begin{array}{l}
Y_{i}^{n+1}=\bar{Y}_{i}+\lambda_{0} T \Delta \tilde{t}\left(Y^{*}\left(\bar{\rho}_{i}, \bar{e}_{i}\right)-Y_{i}^{n+1}\right) \\
\tilde{\varphi}_{i}^{n+1}=\overline{\tilde{\varphi}}_{i}, \quad \tilde{\varphi} \in\{\tilde{\rho}, \tilde{\rho} \tilde{\mathbf{u}}, \tilde{\rho} \tilde{E}\}
\end{array}\right.
$$

Proof of property $(v)$. We define $\tilde{h}=h / L$ where $h$ is the mesh size. The acoustic CFL restriction 20 is very restrictive in low Mach regime as $\Delta \tilde{t}=$ $\mathcal{O}(M \tilde{h})$, while the transport CFL restriction 22 is uniform with respect to the Mach number $\Delta \tilde{t}=\mathcal{O}(\tilde{h})$. Thus the LPS-IMEX $(\theta)$ scheme is stable in the uniform sense with respect to the Mach number $M$, while the $\operatorname{LPS}-\operatorname{EX}(\theta)$ scheme is not. 
Proof of property (iv). In order to evaluate the truncation error in the low Mach regime, we use the classical tool of modified equations. With a slight abuse of notation, we consider $\tilde{\varphi}\left(\mathbf{x}_{i}, t^{n}\right)=\tilde{\varphi}_{i}^{n}$ so that we can substitute these functions in discrete formulas.

We assume that we are in low Mach regime, namely $M \ll 1$ and $\tilde{\nabla} \tilde{p}=\mathcal{O}\left(M^{2}\right)$. This hypothesis yields that for $j \in N(i)$, we have $\tilde{\Pi}_{j}^{n}=\tilde{\Pi}_{i}^{n}+\mathcal{O}\left(M^{2} \tilde{h}\right)$ and $\tilde{\Pi}_{j}^{n+1-}=\tilde{\Pi}_{i}^{n+1-}+\mathcal{O}\left(M^{2} \tilde{h}\right)$ for the discrete unknowns.

The rescaled discretization of the acoustic step $(19)$ is consistent with

$$
\left\{\begin{aligned}
\partial_{\tilde{t}} Y & =\mathcal{O}(\Delta \tilde{t}) \\
\partial_{\tilde{t}} \tilde{\tau}-\tilde{\tau} \tilde{\nabla} \cdot \tilde{\mathbf{u}} & =\mathcal{O}(\Delta \tilde{t})+\mathcal{O}(M \tilde{h}) \\
\partial_{\tilde{t}} \tilde{\mathbf{u}}+\frac{\tilde{\tau}}{M^{2}} \tilde{\nabla} \tilde{p}(\tilde{\rho}, \tilde{e}, Y) & =\mathcal{O}(\Delta \tilde{t})+\mathcal{O}\left(\frac{\theta}{M} \tilde{h}\right) \\
\partial_{\tilde{t}} \tilde{E}+\tilde{\tau} \tilde{\nabla} \cdot(\tilde{p}(\tilde{\rho}, \tilde{e}, Y) \tilde{\mathbf{u}}) & =\mathcal{O}(\Delta \tilde{t})+\mathcal{O}(M \tilde{h})+\mathcal{O}(\theta M \tilde{h})
\end{aligned}\right.
$$

for both the implicit solver (18) and the explicit solver (17).

The rescaled discretization of the transport step 22 is consistent with

$$
\partial_{\tilde{t}} \tilde{\varphi}+\tilde{\mathbf{u}} \cdot \tilde{\nabla} \tilde{\varphi}=\mathcal{O}(\Delta \tilde{t})+\mathcal{O}(\tilde{h})+\mathcal{O}(M \tilde{h}) \quad \text { for } \tilde{\varphi} \in\{\tilde{\rho} Y, \tilde{\rho}, \tilde{\rho} \tilde{\mathbf{u}}, \tilde{\rho} \tilde{E}\}
$$

The rescaled discretization of the phase transition step $(23)$ is consistent with

$$
\begin{cases}\partial_{\tilde{t}}(\tilde{\rho} Y) & =\lambda_{0} T\left(\tilde{\rho} Y^{*}(\tilde{\rho}, \tilde{e})-\tilde{\rho} Y\right)+\mathcal{O}(\Delta \tilde{t}), \\ \partial_{\tilde{t}} \tilde{\varphi} & =\mathcal{O}(\Delta \tilde{t}) \quad \text { for } \tilde{\varphi} \in\{\tilde{\rho}, \tilde{\rho} \tilde{\mathbf{u}}, \tilde{\rho} \tilde{E}\} .\end{cases}
$$

So that the equivalent equation verified by the overall rescaled scheme reads

$$
\left\{\begin{aligned}
\partial_{t}(\rho Y)+\nabla \cdot(\rho Y \mathbf{u}) & =\lambda_{0} \rho\left(Y^{*}(\rho, e)-Y\right)+\mathcal{O}(\Delta \tilde{t})+\mathcal{O}(\tilde{h})+\mathcal{O}(M \tilde{h}), \\
\partial_{t} \rho+\nabla \cdot(\rho \mathbf{u}) & =\mathcal{O}(\Delta \tilde{t})+\mathcal{O}(\tilde{h})+\mathcal{O}(M \tilde{h}), \\
\partial_{t}(\rho \mathbf{u})+\nabla \cdot(\rho \mathbf{u} \otimes \mathbf{u})+\nabla p & =\mathcal{O}(\Delta \tilde{t})+\mathcal{O}\left(\frac{\theta \tilde{h}}{M}\right)+\mathcal{O}(\tilde{h})+\mathcal{O}(M \tilde{h}), \\
\partial_{t}(\rho E)+\nabla \cdot[(\rho E+p) \mathbf{u}] & =\mathcal{O}(\Delta \tilde{t})+\mathcal{O}(\tilde{h})+\mathcal{O}(M \tilde{h})+\mathcal{O}(\theta M \tilde{h}) .
\end{aligned}\right.
$$

As a consequence, provided that we impose the asymptotic behavior $\theta=$ $\mathcal{O}(M)$, the truncation error of scheme (19)-(21)- 23 is uniform with respect to $M$ for both the implicit solver (18) and the explicit solver (17). This concludes the proof of property (iv). 
Let us note that the classical Suliciu relaxation fluxes obtained for $\theta=1$ do not have a truncation error that is uniform with respect to the Mach number.

\section{Numerical results}

We propose to test both LPS-IMEX $(\theta)$ and LPS-EX $(\theta)$ schemes against low Mach number test cases and order 1 Mach number test cases. LPS-EX $(\theta)$ computations are performed with a time step satisfying both (9) and (13), while LPS-IMEX $(\theta)$ computations are performed with a time step defined by an explicit evaluation of (13) (explicit means here that $u^{*}$ defined by (8) is used to evaluate $\Delta t$ ).

So far, the value of the $\theta_{i j}$ parameter has not been specified. Actually, the numerical evidences presented here will focus on the following three choices:

- $\theta=1$, we will simply set $\theta_{i j}=1$.

- $\theta=\mathcal{O}(M)$, we will set $\theta_{i j}=\min \left(M_{i j}^{n}, 1\right)$, where $M_{i j}^{n}=\frac{\left|u_{i j}^{*}\right|}{\max \left(c_{i}, c_{j}\right)}$ is an evaluation of the Mach number at each mesh interface and at time $t^{n}\left(u_{i j}^{*}\right.$ is defined by (8)).

- $\theta=0$, we will simply set $\theta_{i j}=0$.

Let us recall that the classical Suliciu relaxation fluxes correspond to the choice $\theta=1$ and are expected to have too much numerical diffusion in the low Mach regime. The choice $\theta=\mathcal{O}(M)$ is based on a flow sensor $M_{i j}^{n}$ that locally evaluates the Mach number. We expect this value to be sufficiently low to improve the accuracy of the scheme in the low Mach regime, see properties (iv) of theorem 2 and 3 . The case $\theta=0$ corresponds to a centered discretization of the pressure gradient in the Lagrange step and is used to show the robustness of the numerical scheme with respect to the choice of $\theta_{i j}$. Let us note that one may choose any value $0 \leq \theta_{i j}<1$ to obtain different numerical fluxes having less numerical diffusion than the classical Suliciu relaxation fluxes.

For the sake of reproducibility, we shall use a simplified two-phase thermodynamic mixture model: we consider a mixture of two perfect gases with different adiabatic coefficients $\gamma_{1}>\gamma_{2}>1$. We refer for instance the reader to [6] and the references therein. We assume that $\lambda_{0} \rightarrow \infty$, so that the thermodynamic equilibrium is instantaneously achieved. The mass fraction, pressure and sound 
speed of the mixture are given by

$$
\left(Y^{*}, p^{\mathrm{HEM}}, c^{2}\right)(\rho, e)=\left\{\begin{array}{cl}
\left(1,\left(\gamma_{1}-1\right) \rho e, \gamma_{1}\left(\gamma_{1}-1\right) e\right) & \text { if } \rho<\rho_{1}^{*}, \\
\left(\left(\frac{\rho_{1}^{*}}{\rho}\right)\left(\frac{\rho-\rho_{2}^{*}}{\rho_{1}^{*}-\rho_{2}^{*}}\right),\left(\gamma_{1}-1\right) \rho_{1}^{*} e,\left(\gamma_{1}-1\right)^{2}\left(\frac{\rho_{1}^{*}}{\rho}\right)^{2} e\right) & \text { if } \rho_{1}^{*} \leq \rho \leq \rho_{2}^{*}, \\
\left(0,\left(\gamma_{2}-1\right) \rho e, \gamma_{2}\left(\gamma_{2}-1\right) e\right) & \text { if } \rho_{2}^{*}<\rho,
\end{array}\right.
$$

where

$$
\rho_{1}^{*}=\frac{1}{\exp (1)}\left(\frac{\gamma_{2}-1}{\gamma_{1}-1}\right)^{\frac{\gamma_{2}}{\gamma_{2}-\gamma_{1}}}, \quad \rho_{2}^{*}=\frac{1}{\exp (1)}\left(\frac{\gamma_{2}-1}{\gamma_{1}-1}\right)^{\frac{\gamma_{1}}{\gamma_{2}-\gamma_{1}}}
$$

Unless otherwise stated, we consider $\gamma_{1}=2, \gamma_{2}=1.4$, which gives $\rho_{1}^{*} \simeq$ 3.1205576, $\rho_{2}^{*} \simeq 7.801394$. Figure (2) displays the sound speed as a function of the density for $e=1000$. We observe a large decrease of the sound speed when mixture occurs. This behavior is quite common in mixture equation of state and will induce a rise of the Mach number, so that the ability to simulate a wide range of flow regimes is of utmost importance for a numerical method to simulate homogeneous models for two phase flows.

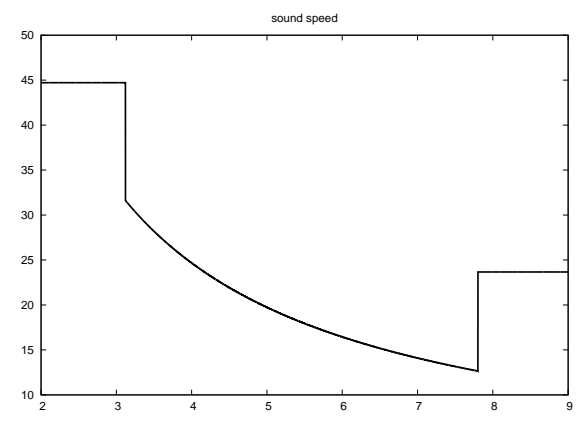

Figure 2: Mixture of two perfect gases with different adiabatic coefficients $\left(\gamma_{1}=2, \gamma_{2}=1.4\right)$. Sound speed as a function of the density for $e=1000$.

\subsection{Low Mach number examples}

We consider low Mach test cases and try to examine two questions : the accuracy gain for simulations on coarse grid in the low Mach regime using $\theta=\mathcal{O}(M)$ or $\theta=0$ instead of $\theta=1$, then the benefit of using a semi-implicit strategy in terms of CPU time. 
Bubble in a vortex test case $\left(10^{-4} \leq M \leq 10^{-1}\right)$. The computational domain is $\Omega=[0,1]^{2}$. The initial condition is given by

$$
\left\{\begin{array}{l}
p(x, y, t=0)=1000, \\
\rho(x, y, t=0)= \begin{cases}1 & \text { if }(x-0.5)^{2}+(y-0.25)^{2} \leq 0.01, \\
10 & \text { if }(x-0.5)^{2}+(y-0.25)^{2}>0.01,\end{cases} \\
Y(x, y, t=0)= \begin{cases}1 & \text { if }(x-0.5)^{2}+(y-0.25)^{2} \leq 0.01, \\
0 & \text { if }(x-0.5)^{2}+(y-0.25)^{2}>0.01,\end{cases} \\
u(x, y, t=0)=2 \sin ^{2}(\pi x) \sin (\pi y) \cos (\pi y), \\
v(x, y, t=0)=-2 \sin (\pi x) \cos (\pi x) \sin ^{2}(\pi y) .
\end{array}\right.
$$

We impose no-slip boundary conditions. The Mach number for the resulting flows is of order $10^{-4}$ in phase $1(Y=1)$ and $10^{-3}$ in phase $2(Y=0)$ so that pure phases are in the low Mach regime. Nevertheless, since the sound speed of the mixture is smaller than the sound speed of pure phase, we observe a Mach number that goes up to $10^{-1}$ in the mixture. We plot the solution at time $t=0.5 s$.

Figure (3)-(5) display the results obtained with the LPS-EX $(\theta)$ scheme for $\theta=1$ and $\theta=\mathcal{O}(M)$. We use as a reference solution an approximation computed with LPS-EX $(\theta=1)$ using a $1.6 \times 10^{5}$-cell triangular mesh. The choice $\theta_{i j}=\min \left(M_{i j}^{n}, 1\right)$ leads to approximations that are much more accurate than $\theta_{i j}=1$. On figures (4)-(5), we obtain similar results for the $\operatorname{LPS}-\operatorname{IMEX}(\theta)$ scheme. The LPS-IMEX $(\theta)$ and $\operatorname{LPS}-\operatorname{EX}(\theta)$ schemes for $\theta=\mathcal{O}(M)$ require respectively $2479 \mathrm{~s}=41 \mathrm{~min} 19 \mathrm{~s}$ and $16465 \mathrm{~s}=4 \mathrm{~h} 34 \mathrm{~min} 25 \mathrm{~s}$ of $\mathrm{CPU}$ time, so that using an implicit solver for the acoustic step is 6.6 times faster.

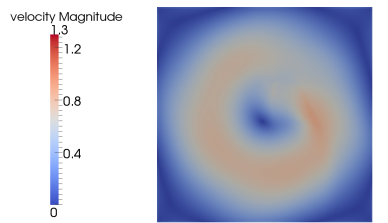

(a)

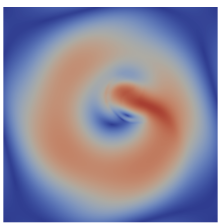

(b)

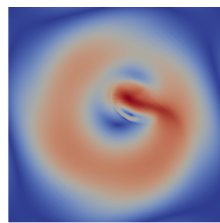

$(c)$

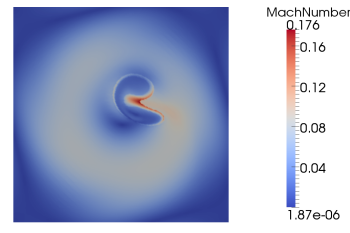

$(d)$

Figure 3: Bubble in a vortex test case. Profile at time $\mathrm{t}=0.5 \mathrm{~s}$ of the velocity magnitude for (a) $\operatorname{LSP}-\operatorname{EX}(\theta=1)$, (b) $\operatorname{LSP}-\operatorname{EX}(\theta=\mathcal{O}(M))$ with a $200 \times 200$-cell Cartesian mesh, (c) velocity magnitude obtained with the reference solution and (d) Mach number obtained with the reference solution.

Subsonic flow in a channel with bump $\left(10^{-3} \leq M \leq 10^{-2}\right)$. We 

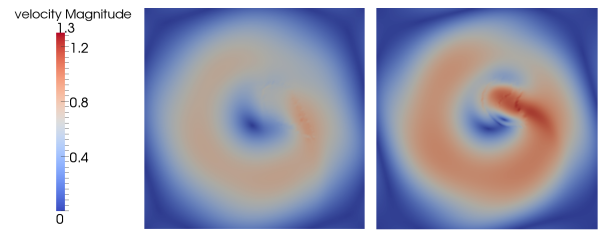

Figure 4: Bubble in a vortex test case. Profile at time $\mathrm{t}=0.5 \mathrm{~s}$ of the velocity magnitude for the $\operatorname{LSP}-\operatorname{IMEX}(\theta=1)$ scheme (left) and the $\operatorname{LSP}-\operatorname{IMEX}(\theta=\mathcal{O}(M))$ scheme (right) on a $200 \times 200$-cell Cartesian mesh. To be compared with (a), (b) and (c) in figure 3

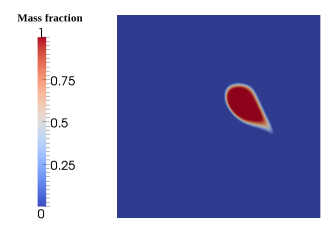

$(a)$

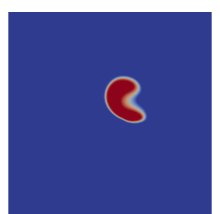

(b)

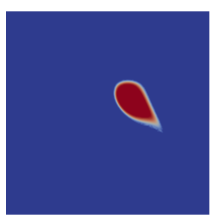

$(c)$

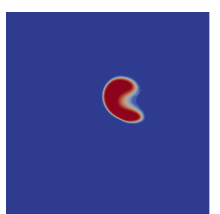

$(d)$

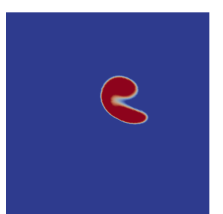

$(e)$

Figure 5: Bubble in a vortex test case. Profile at time $\mathrm{t}=0.5 \mathrm{~s}$ of the mass fraction for (a) $\operatorname{LSP}-\operatorname{EX}(\theta=1)$, (b) $\operatorname{LSP}-\operatorname{EX}(\theta=\mathcal{O}(M))$, (c) $\operatorname{LSP}-\operatorname{IMEX}(\theta=1)$, (d) $\operatorname{LSP}-\operatorname{IMEX}(\theta=\mathcal{O}(M))$ with a $200 \times 200$-cell Cartesian mesh and (e) mass fraction of the reference solution.

consider now the case of a subsonic flow passing a channel with a $20 \%$ sinusoidal bump (see figure 6). A similar test was proposed by [5]. We propose here an adapted version with our simplified EOS for the sake of reproducibility.

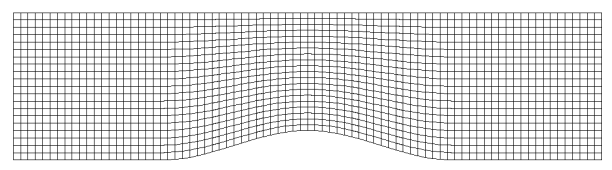

Figure 6: $80 \times 20$ quadrangular mesh of a $20 \%$ sinusoidal bump.

The initial condition is given by

$$
(\rho, Y, p, u, v)(x, y, t=0)=\left(7.81,0,3124, u_{i n}, 0\right)
$$

We impose an inlet boundary condition at $0 \times[0,1]:(e+p / \rho, u, v)=\left(1400, u_{i n}, 0\right)$ and an outlet boundary condition at $4 \times[0,1]: p=3124$. Wall boundary conditions are set on the other boundaries. At the inflow, the fluid is composed of pure phase 2 which is very close to the saturation state. As the pressure drops down with the section restriction, a small concentration of phase 1 appears in- 
ducing a large change of the Mach number. The flow regime is determined by the choice of $u_{i n}>0$. We consider two configurations, $u_{i n}=0.2$ leading to a subsonic flow, while $u_{i n}=12$ leads to a transonic flow and will be used in the next paragraph. We let the flow evolves until it converges to a stationary state. All tests are performed on a $80 \times 20$ quadrangular mesh.

Figures 7 and 8 display the flow profile at $t=5 \mathrm{~s}$, we observe that LPS-EX $(\theta)$ and LPS-IMEX $(\theta)$ schemes are more diffusive for $\theta=1$ than for $\theta=\mathcal{O}(M)$ or $\theta=0$. In terms of CPU time, for $\theta=\mathcal{O}(M)$, we observe that the $\operatorname{LPS}-\operatorname{IMEX}(\theta)$ scheme is 79.2 times faster than the LPS-EX $(\theta)$ scheme thanks to the use of a material velocity CFL condition (13).

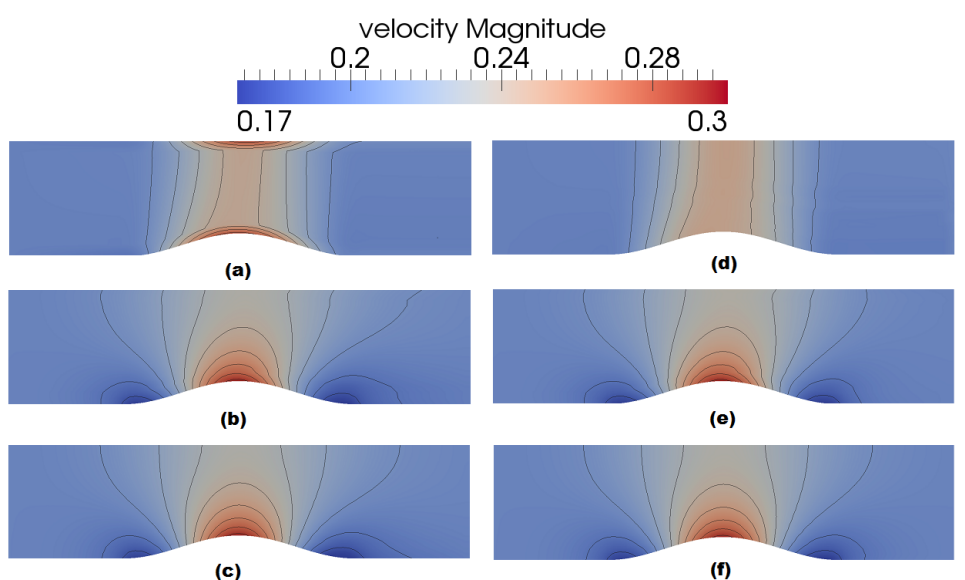

Figure 7: Subsonic flow in a channel with bump. Profile at $\mathrm{t}=5 \mathrm{~s}$ of the velocity magnitude for (a) $\operatorname{LPS}-\operatorname{EX}(\theta=1)$, (b) LPS-EX $(\theta=\mathcal{O}(M))$, (c) $\operatorname{LPS}-\operatorname{EX}(\theta=0)$, (d) $\operatorname{LPS}-\operatorname{IMEX}(\theta=1)$, (e) LPS-IMEX $(\theta=\mathcal{O}(M))$, (f) LPS-IMEX $(\theta=0)$ using a $80 \times 20$ quadrangular mesh.

\subsection{Compressible flow examples}

In this section, we assess the ability of our operator splitting scheme to handle cases where the flow is not in the low Mach regime over the whole computational domain. This is an important issue since the modification introduced by $\theta$ modifies the numerical diffusion of the scheme, which is relevant in the low Mach number regime, but might give rise to instabilities when the Mach number is of order 1 or larger. We will see that even with a centered pressure discretization, ie. for $\theta=0$, the solution remains stable. 


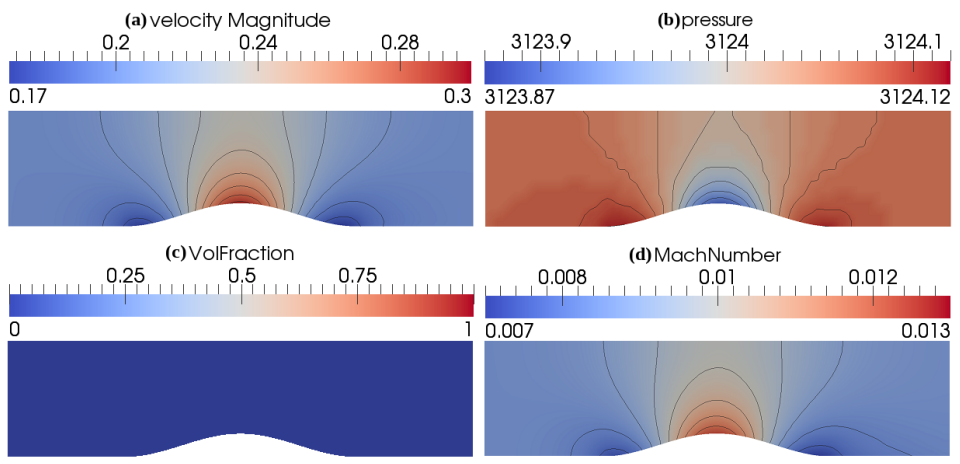

Figure 8: Subsonic flow in a channel with bump. Profile at $\mathrm{t}=5 \mathrm{~s}$ of the (a) velocity magnitude, (b) pressure, (c) mass fraction and (d) Mach number for the LPS-IMEX $(\theta=0)$ ) using a $80 \times 20$ quadrangular mesh.

Two-rarefaction Riemann problem with appearance of phase $1(1 \leq$ $M)$. The computational domain is $\Omega=[0,1]$. The adiabatic coefficients are $\gamma_{1}=1.6, \gamma_{2}=1.4$, which yields $\rho_{1}^{*} \simeq 6.2855651, \rho_{2}^{*} \simeq 9.4283477$. The initial condition is given by

$$
(\rho, Y, p, u)(x, t=0)= \begin{cases}(10,0,1,-2), & \text { for } x<0.5 \\ (10,0,1,1), & \text { for } x>0.5\end{cases}
$$

We impose Neumann boundary conditions and plot the solution at time $t=0.1 \mathrm{~s}$. For the initial condition, the medium is composed by pure phase 2 only, but an intermediate zone with pure phase 1 appears for $t>0$.

Figure (9) displays the results obtained with LPS-EX $(\theta)$ and $\operatorname{LPS}-\operatorname{IMEX}(\theta)$ schemes for $\theta=1$ and $\theta=0$. We use as a reference solution an approximation computed with LPS-EX $(\theta=1)$ using a 10 000-cell mesh. All schemes show a good agreement with the reference solution. The LPS-EX $(\theta=0)$ and LPS$\operatorname{IMEX}(\theta=0)$ schemes are slightly less diffusive than the $\operatorname{LPS}-\operatorname{EX}(\theta=1)$ and $\operatorname{LPS} \operatorname{IMEX}(\theta=1)$ schemes. Let us underline that despite part of the solutions clearly do not belong to the low Mach regime since $M \approx 5.4$ in the left part of the domain and $M \approx 2.7$ in the right part of the domain, the schemes LPS$\operatorname{EX}(\theta=0)$ and $\operatorname{LPS}-\operatorname{IMEX}(\theta=0)$ are stable and provide good numerical results while involving a centered pressure discretization with $\theta_{i j}=0$.

Remark 8. The numerical diffusion of the mass fraction generates a mixture zone where the sound speed is much smaller than in pure phases. This leads to 
an overshot for the Mach number value to be observed in figure 9 .

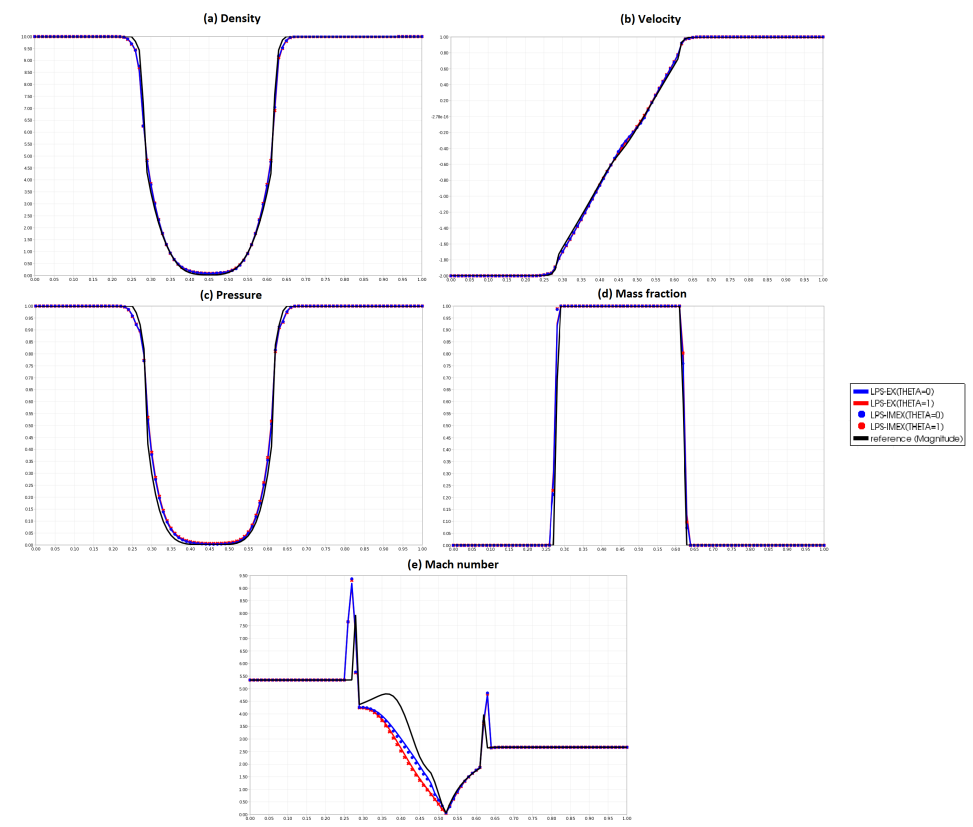

Figure 9: Two-rarefaction Riemann problem with appearance of phase 1. Profile at $t=0.1$ $\mathrm{s}$ of the (a) density, (b) velocity magnitude, (c) pressure, (d) mass fraction and (e) Mach number for the LPS-EX $(\theta=1), \operatorname{LPS}-\operatorname{EX}(\theta=0), \operatorname{LPS}-\operatorname{IMEX}(\theta=1), \operatorname{LPS}-\operatorname{IMEX}(\theta=0)$ using a 1000-cell grid, together with reference solution.

Transonic flow in a channel with bump $\left(10^{-1} \leq M \leq 1.12\right)$. We consider now the case of a transonic flow passing a channel with a $20 \%$ sinusoidal bump, see figure 6. The initial and boundary conditions were presented in the previous section for a subsonic flow in a channel with bump. We consider here the configuration $u_{i n}=12$ that leads to a transonic flow.

Figure 10 and 11 display the results obtained with LPS-EX $(\theta)$ and LPS$\operatorname{IMEX}(\theta)$ schemes for $\theta=1, \theta=\mathcal{O}(M)$ and $\theta=0$. All schemes are able to compute accurate approximate solutions. The results obtained with $\theta=0$ are slightly less diffused than the results obtained with $\theta=1$ and $\theta=\mathcal{O}(M)$.

\section{Conclusion}

We proposed an operator splitting discretization strategy for a HRM and a HEM two-phase flow model with mass transfer and velocity equilibrium. The 


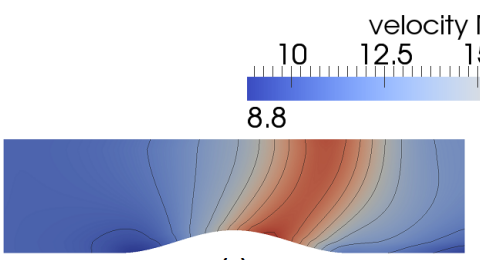

(a)

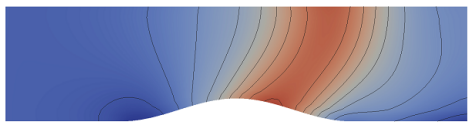

(b)

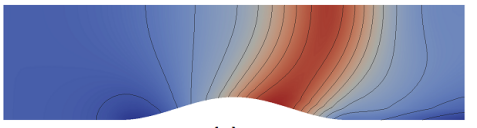

(c)
22

(d)

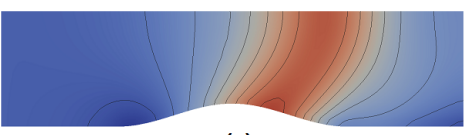

(e)

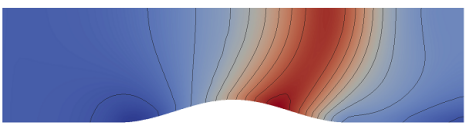

(f)

Figure 10: Transonic flow in a channel with bump. Profile at $\mathrm{t}=5 \mathrm{~s}$ of the velocity magnitude for (a) $\operatorname{LPS}-\operatorname{EX}(\theta=1)$, (b) LPS-EX $(\theta=\mathcal{O}(M))$, (c) $\operatorname{LPS}-\operatorname{EX}(\theta=0)$, (d) $\operatorname{LPS}-\operatorname{IMEX}(\theta=1)$, (e) $\operatorname{LPS}-\operatorname{IMEX}(\theta=\mathcal{O}(M))$, (f) LPS-IMEX $(\theta=0)$ using a $80 \times 20$ quadrangular mesh.

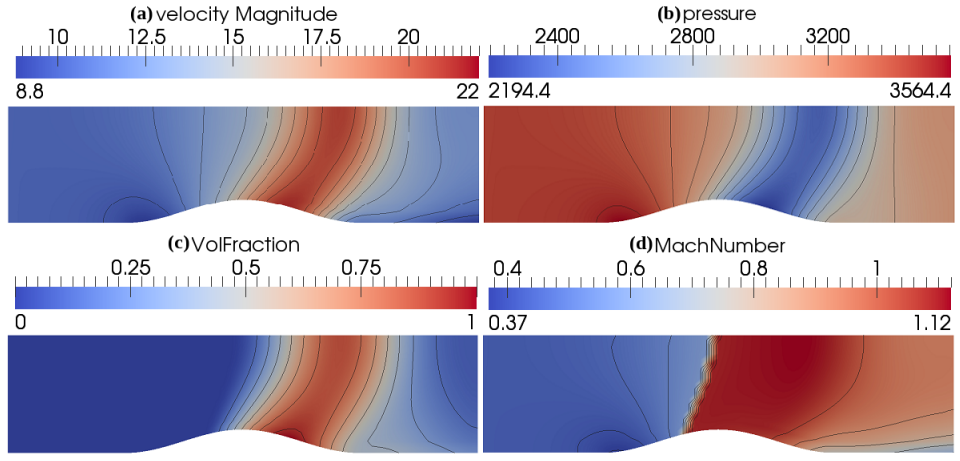

Figure 11: Transonic flow in a channel with bump. Profile at $t=5 \mathrm{~s}$ of the (a) velocity magnitude, (b) pressure, (c) mass fraction and (d) Mach number for LPS-EX( $\theta=0)$ using a $80 \times 20$ quadrangular mesh. 
splitting decouples the transport, acoustic and mass transfer phenomena. The overall algorithm is conservative with respect to the global mass, global momentum and global total energy. The scheme allows the use of unstructured two-dimensional meshes and for one-dimensional problems, the treatment of the convective part boils down to a classical Lagrange-Remap procedure. A first numerical scheme using an explicit time update was proposed. Under a classical CFL condition, this numerical scheme enjoys stability properties: positivity of the global mass, the mass fraction and satisfies a discrete entropy inequality.

Considering one-dimensional problems, we performed an analysis of the truncation error to shed light on its dependency on the Mach number $M$ in the low Mach regime. In order to obtain a numerical scheme whose truncation error is uniform with respect to $M$ in the low Mach regime, we proposed a simple modification of the numerical flux in the acoustic step. It is possible to show that this modified numerical scheme can be obtained equivalently thanks to an approximate Riemann solver that is consistent in the integral sense. As a result, the modified numerical scheme is shown to enjoy the same stability properties as the first splitting scheme under the same CFL conditions.

We also proposed a semi-implicit version of the algorithm that allows to obtain a numerical scheme that is stable under a CFL conditions that involves only the material velocity and with a truncation error that is uniform with respect to $M$.

The present work deals with simple two-phase flows in an academic setting. Further developments using the HRM and HEM models will consider a drift velocity, friction and energy source terms and gravity. Finally, as far as more complex two-phase flows models are concerned, a Lagrange-Remap like algorithm using the same kind of operator splitting has already been proposed for the Baer-Nunziato system in [31]. The study of the low Mach behavior of this numerical scheme has to be performed. Since the Baer-Nunziato model comprises several material and sound velocities, this task is not a straightforward extension of the analysis presented in the present paper for the HRM and HEM models. 


\section{Appendix A. Approximate Riemann solver for the modified acoustic scheme}

In this section, we consider only one-dimensional problems and denote $x \in \mathbb{R}$, the space variable. The numerical scheme (7)-(8) belongs to the category of fluxbased solver. Indeed, this solver relies on an update formula (7) that involves the modified fluxes (8). We will prove in this section that this modified flux solver can also be obtained thanks to an approximate Riemann solver in the sense of Harten, Lax and Van Leer [32, that is consistent with the integral form of the system

$$
\partial_{t} \mathbf{W}+\partial_{m} \mathbf{F}(\mathbf{W})=0,
$$

where $\mathbf{W}=(Y, \tau, u, v, E, \Pi)^{T}, \mathbf{F}(\mathbf{W})=\left(0,-u, \Pi, 0, \Pi u, a^{2} u\right)^{T}$, and the mass variable $m$ is defined by $\mathrm{d} m=\rho\left(x, t^{n}\right) \mathrm{d} x$. This property is crucial in the following and is very easy to use in order to establish the forthcoming stability results.

Remark 9. It is interesting to note that the corresponding approximate Riemann solver is not unique. In fact, for any given numerical flux $\mathbf{F}^{\theta}\left(\mathbf{W}_{L}, \mathbf{W}_{R}\right)$, we are able to construct an infinity of approximate Riemann solvers in the sense of Harten, Lax and Van Leer, that are consistent with the integral form of A.1 and such that the flux coincides with $\mathbf{F}^{\theta}\left(\mathbf{W}_{L}, \mathbf{W}_{R}\right)$. We refer to the proof of proposition 1 below, where the corresponding approximate Riemann solvers depend on the value of the constant parameter $a>0$.

If $\Delta t>0$ and $\Delta x_{j}>0$ denote respectively the time and space step of cell $j$, we set $\Delta m_{j}=\rho_{j}^{n} \Delta x_{j}$ and we consider the one-dimensional scheme

$$
\left\{\begin{aligned}
\mathbf{W}_{j}^{n+1-} & =\mathbf{W}_{j}^{n}-\frac{\Delta t}{\Delta m_{j}}\left(\mathbf{F}_{j+1 / 2}-\mathbf{F}_{j-1 / 2}\right), \\
\mathbf{F}_{j+1 / 2} & =\mathbf{F}^{\theta}\left(\mathbf{W}_{j}^{n}, \mathbf{W}_{j+1}^{n}\right), \\
\mathbf{F}^{\theta}\left(\mathbf{W}_{L}, \mathbf{W}_{R}\right) & =\left(0,-u^{*}, \Pi^{*, \theta}, 0, \Pi^{*, \theta} u^{*}, a^{2} u^{*}\right)^{T},
\end{aligned}\right.
$$

where $u^{*}$ and $\Pi^{*, \theta}$ are given by

$$
\left\{\begin{aligned}
u^{*} & =\frac{\left(u_{R}+u_{L}\right)}{2}-\frac{1}{2 a}\left(\Pi_{R}-\Pi_{L}\right), \\
\Pi^{*, \theta} & =\frac{\left(\Pi_{R}+\Pi_{L}\right)}{2}-\theta \frac{a}{2}\left(u_{R}-u_{L}\right) .
\end{aligned}\right.
$$

This one-dimensional scheme allows to recover scheme (7)-(8) by taking for $u$ 
(resp. $v$ ) the normal velocity (resp. the tangential velocity) at the face where we estimate the numerical flux. Besides we have the following property :

Proposition 1. There exists a simple approximate Riemann solver that is an approximation of the Riemann problem associated with system A.1 and whose associated flux matches the flux of the modified acoustic solver A.2-A.3. More precisely, there exists a self-similar function

$\mathbf{W}_{R P}^{\theta}\left(\frac{m}{t} ; \mathbf{W}_{L}, \mathbf{W}_{R}\right)=(Y, \tau, u, v, E, \Pi)\left(\frac{m}{t} ; \mathbf{W}_{L}, \mathbf{W}_{R}\right)= \begin{cases}\mathbf{W}_{L}, & \text { if } m / t<-a, \\ \mathbf{W}_{L}^{*, \theta}, & \text { if }-a \leq m / t<0, \\ \mathbf{W}_{R}^{*, \theta}, & \text { if } 0 \leq m / t<+a, \\ \mathbf{W}_{R}, & \text { if }+a \leq m / t .\end{cases}$

such that

$$
\begin{aligned}
\mathbf{F}^{\theta}\left(\mathbf{W}_{R}, \mathbf{W}_{L}\right) & =\mathbf{F}\left(\mathbf{W}_{L}\right)-\int_{-\infty}^{0}\left[\mathbf{W}_{R P}^{\theta}\left(\xi ; \mathbf{W}_{L}, \mathbf{W}_{R}\right)-\mathbf{W}_{L}\right] \mathrm{d} \xi \\
& =\mathbf{F}\left(\mathbf{W}_{R}\right)+\int_{0}^{+\infty}\left[\mathbf{W}_{R P}^{\theta}\left(\xi ; \mathbf{W}_{L}, \mathbf{W}_{R}\right)-\mathbf{W}_{R}\right] \mathrm{d} \xi \\
& =\frac{1}{2}\left(\mathbf{F}\left(\mathbf{W}_{L}\right)+\mathbf{F}\left(\mathbf{W}_{R}\right)\right)-\frac{a}{2}\left(\mathbf{W}_{L}^{*, \theta}-\mathbf{W}_{L}\right)-\frac{a}{2}\left(\mathbf{W}_{R}-\mathbf{W}_{R}^{*, \theta}\right) .
\end{aligned}
$$

The states $\mathbf{W}_{L}^{*, \theta}=\left(Y_{L}^{*, \theta}, \tau_{L}^{*, \theta}, u_{L}^{*, \theta}, v_{L}^{*, \theta}, E_{L}^{*, \theta}, \Pi_{L}^{*, \theta}\right)^{T}$ and $\mathbf{W}_{R}^{*, \theta}=\left(Y_{R}^{*, \theta}, \tau_{R}^{*, \theta}, u_{R}^{*, \theta}, v_{R}^{*, \theta}, E_{R}^{*, \theta}, \Pi_{R}^{*, \theta}\right)^{T}$ are given by

$$
\begin{aligned}
Y_{L}^{*, \theta} & =Y_{L}, & Y_{R}^{*, \theta} & =Y_{R}, \\
\tau_{L}^{*, \theta} & =\tau_{L}+\frac{1}{a}\left(u^{*}-u_{L}\right), & \tau_{R}^{*, \theta} & =\tau_{R}+\frac{1}{a}\left(u_{R}-u^{*}\right), \\
u_{L}^{*, \theta} & =u^{*}+\frac{1}{2}(\theta-1)\left(u_{R}-u_{L}\right), & u_{R}^{*, \theta} & =u^{*}+\frac{1}{2}(1-\theta)\left(u_{R}-u_{L}\right), \\
v_{L}^{*, \theta} & =v_{L}, & v_{R}^{*, \theta} & =v_{R}, \\
E_{L}^{*, \theta} & =E_{L}+\frac{1}{a}\left(\Pi_{L} u_{L}-\Pi^{*, \theta} u^{*}\right), & E_{R}^{*, \theta} & =E_{R}+\frac{1}{a}\left(\Pi^{*, \theta} u^{*}-\Pi_{R} u_{R}\right) \\
\Pi_{L}^{*, \theta} & =\Pi^{*, \theta}=1, & \Pi_{R}^{*, \theta} & =\Pi^{*, \theta}=1 .
\end{aligned}
$$

We define $\Pi^{*}=\Pi_{R}^{*, \theta}=\Pi_{L}^{*, \theta},\left(Y_{L}^{*}, \tau_{L}^{*}, v_{L}^{*}\right)=\left(Y_{L}^{*, \theta}, \tau_{L}^{*, \theta}, v_{L}^{*, \theta}\right)$ and $\left(Y_{R}^{*}, \tau_{R}^{*}, v_{R}^{*}\right)=$ $\left(Y_{R}^{*, \theta}, \tau_{R}^{*, \theta}, v_{R}^{*, \theta}\right)$ that are independant of $\theta$.

Proof. $\mathbf{W}_{\mathrm{RP}}^{\theta}$ is consistent with the integral form of the system A.1, if for a 
given $\mathbf{W}_{L}$ and $\mathbf{W}_{R}$, we have : $\mathbf{F}\left(\mathbf{W}_{R}\right)-\mathbf{F}\left(\mathbf{W}_{L}\right)=-a\left(\mathbf{W}_{L}^{*, \theta}-\mathbf{W}_{L}\right)+a\left(\mathbf{W}_{R}-\right.$ $\left.\mathbf{W}_{R}^{*, \theta}\right)$, which reads

$$
\mathbf{W}_{R}^{*, \theta}+\mathbf{W}_{L}^{*, \theta}=\mathbf{W}_{R}+\mathbf{W}_{L}-\frac{1}{a}\left(\mathbf{F}\left(\mathbf{W}_{R}\right)-\mathbf{F}\left(\mathbf{W}_{L}\right)\right)
$$

If the resulting flux of this approximate Riemann solver is $F^{\theta}\left(\mathbf{W}_{L}, \mathbf{W}_{R}\right)$, then A.5 is verified and yields

$$
2 F^{\theta}\left(\mathbf{W}_{L}, \mathbf{W}_{R}\right)=\mathbf{F}\left(\mathbf{W}_{R}\right)+\mathbf{F}\left(\mathbf{W}_{L}\right)-a\left(\mathbf{W}_{L}^{*, \theta}-\mathbf{W}_{L}\right)-a\left(\mathbf{W}_{R}-\mathbf{W}_{R}^{*, \theta}\right)
$$

or equivalently

$$
\mathbf{W}_{R}^{*, \theta}-\mathbf{W}_{L}^{*, \theta}=\mathbf{W}_{R}-\mathbf{W}_{L}+\frac{1}{a}\left(2 F^{\theta}\left(\mathbf{W}_{L}, \mathbf{W}_{R}\right)-\mathbf{F}\left(\mathbf{W}_{L}\right)-\mathbf{F}\left(\mathbf{W}_{R}\right)\right) .
$$

Both (A.7) and (A.8) provide

$\mathbf{W}_{L}^{*, \theta}=\mathbf{W}_{L}-\frac{1}{a}\left(F^{\theta}\left(\mathbf{W}_{L}, \mathbf{W}_{R}\right)-\mathbf{F}\left(\mathbf{W}_{L}\right)\right), \quad \mathbf{W}_{R}^{*, \theta}=\mathbf{W}_{R}+\frac{1}{a}\left(F^{\theta}\left(\mathbf{W}_{L}, \mathbf{W}_{R}\right)-\mathbf{F}\left(\mathbf{W}_{R}\right)\right)$.

This definition of $\mathbf{W}_{L}^{*, \theta}$ and $\mathbf{W}_{R}^{*, \theta}$ as a function of $\mathbf{W}_{L}$ and $\mathbf{W}_{R}$ corresponds to A.6 and verifies A.7 and A.8. This yields the desired results.

Using this approximate Riemann solver, we deduce that the modified acoustic solver A.2 is stable under the CFL condition

$$
2 a \Delta t \leq \Delta m_{j}
$$

that does not depend on the modification $\theta$. Moreover, when $\theta=1$ the selfsimilar function $\mathbf{W}_{R P}^{\theta}$ defined in proposition 1 degenerates to the exact solution of the Riemann problem associated with the system A.1.

If one takes into account the equilibrium projection step of the relaxation strategy into the approximate Riemann solver of proposition 1 we have $\Pi_{L}=$ $p\left(\tau_{L}, e_{L}, Y_{L}\right)$, and $\Pi_{R}=p\left(\tau_{R}, e_{R}, Y_{R}\right)$. Under this assumption, it is easy to check that the first coordinates $(Y, \tau, u, v, E)$ of the self-similar function $\mathbf{W}_{\mathrm{RP}}^{\theta}$ are consistent with the integral form of the system

$$
\partial_{t} \mathbf{V}+\partial_{m} \mathbf{F}(\mathbf{V})=0
$$

where $\mathbf{V}=(Y, \tau, u, v, E)^{T}$ et $\mathbf{F}(\mathbf{V})=(0,-u, p, 0, p u)^{T}$. 
Finally, the numerical scheme (7)-(8) may be rewritten using intermediate states of the approximate Riemann solver of property 1 under the form

$$
\mathbf{W}_{i}^{n+1-}=\left(1-\tau_{i}^{n} \Delta t \sum_{j \in N(i)} \sigma_{i j} a_{i j}\right) \mathbf{W}_{i}^{n}+\tau_{i}^{n} \Delta t \sum_{j \in N(i)} \sigma_{i j} a_{i j} \mathbf{W}_{i j}^{n, *, \theta}
$$

where $\mathbf{W}_{i j}^{n, *, \theta}=\mathbf{W}_{L}^{*, \theta}$ is given by A.6 for $\mathbf{W}_{L}=\mathbf{W}_{i}^{n}$ and $\mathbf{W}_{R}=\mathbf{W}_{j}^{n}$, with $u=\mathbf{n}_{i j}^{T} \mathbf{u}$ and $v=\left\|\mathbf{u}-\left(\mathbf{n}_{i j}^{T} \mathbf{u}\right) \mathbf{n}_{i j}\right\|$. This form of the scheme will be usefull to study stability property of the acoustic solver. We prove in particular a discrete entropy inequality in the next section.

\section{Appendix B. Discrete entropy inequality}

In this section, we prove a discrete entropy inequality for the LPS-EX $(\theta)$ scheme. We consider the HRM system (1). We denote $\tau=1 / \rho$ and $s$ the specific entropy. We assume as given a mixture equation of state $(\tau, s, Y) \mapsto e^{\mathrm{HRM}}$ that verifies

$$
\partial_{\tau} e^{\mathrm{HRM}}<0, \quad \partial_{s} e^{\mathrm{HRM}}>0, \quad \partial_{\tau \tau} e^{\mathrm{HRM}}>0 .
$$

The mixture entropy $s=s^{\mathrm{HRM}}(\tau, e, Y)$ verifies $e=e^{\mathrm{HRM}}(\tau, s, Y)$, we define the pressure $p^{\mathrm{HRM}}=-\partial_{\tau} e^{\mathrm{HRM}}$ and the sound speed of the mixture $c=$ $\tau \sqrt{\partial_{\tau \tau} e^{\mathrm{HRM}}}$. We also assume that

$$
(\tau, e, Y) \mapsto-s^{\mathrm{HRM}} \text { is convex. }
$$

Finally, as $Y^{*}(\rho, e)$ corresponds to the thermodynamic equilibrium, we assume that function $Y \mapsto s^{\mathrm{HRM}}(\tau, e, Y)$ is minimal at $Y=Y^{*}(\rho, e)$.

In the sequel, $I\left(b, b^{\prime}\right) \subset \mathbb{R}$ denotes the interval between $b \in \mathbb{R}$ and $b^{\prime} \in \mathbb{R}$. We consider the sub-caracteristic condition

$$
\begin{aligned}
& \tau_{L}^{*}>0, \quad-\partial_{\tau} p^{\mathrm{HRM}}\left(\tau, s_{L}, Y_{L}\right) \leq a^{2}, \quad \forall \tau \in I\left(\tau_{L}, \tau_{L}^{*}\right), \\
& \tau_{R}^{*}>0, \quad-\partial_{\tau} p^{\mathrm{HRM}}\left(\tau, s_{R}, Y_{R}\right) \leq a^{2}, \quad \forall \tau \in I\left(\tau_{R}, \tau_{R}^{*}\right),
\end{aligned}
$$

and we begin by proving two technical results.

Lemma 1. We consider for $\theta=1$ the approximate Riemann solver of property 1. whose intermediate states are given by (A.6). Suppose that (B.3) is verified. Let $s_{k}=s^{H R M}\left(\tau_{k}, e_{k}, Y_{k}\right), k=L, R$ and $e_{k}^{*}=E_{k}^{*, \theta=1}-\left(u_{k}^{*, \theta=1}\right)^{2} / 2-\left(v_{k}^{*}\right)^{2} / 2$, 
we have

$$
e_{k}^{*}-e^{H R M}\left(\tau_{k}^{*}, s_{k}, Y_{k}\right)-\frac{\left(p^{H R M}\left(\tau_{k}^{*}, s_{k}, Y_{k}\right)-\Pi^{*}\right)^{2}}{2 a^{2}} \geq 0 .
$$

Proof. We consider the case $k=R$ and let $\tau \in I\left(\tau_{R}, \tau_{R}^{*}\right)$

$$
\begin{aligned}
\phi(\tau)=e^{\mathrm{HRM}}\left(\tau, s_{R}, Y_{R}\right) & -\frac{p^{\mathrm{HRM}}\left(\tau, s_{R}, Y_{R}\right)^{2}}{2 a^{2}}-e^{\mathrm{HRM}}\left(\tau_{R}^{*}, s_{R}, Y_{R}\right)+\frac{p^{\mathrm{HRM}}\left(\tau_{R}^{*}, s_{R}, Y_{R}\right)^{2}}{2 a^{2}} \\
& +p^{\mathrm{HRM}}\left(\tau_{R}^{*}, s_{R}, Y_{R}\right)\left(\tau+\frac{p^{\mathrm{HRM}}\left(\tau, s_{R}, Y_{R}\right)}{a^{2}}-\tau_{R}^{*}-\frac{p^{\mathrm{HRM}}\left(\tau_{R}^{*}, s_{R}, Y_{R}\right)}{a^{2}}\right) .
\end{aligned}
$$

We have $\phi^{\prime}(\tau)=\left(p^{\operatorname{HRM}}\left(\tau, s_{R}, Y_{R}\right)-p^{\operatorname{HRM}}\left(\tau_{R}^{*}, s_{R}, Y_{R}\right)\right)\left(1-\rho^{2} c^{2}\left(\tau, s_{R}, Y_{R}\right) / a^{2}\right)$. If $\tau_{R}>\tau>\tau_{R}^{*}$ (resp. $\tau_{R}<\tau<\tau_{R}^{*}$ ), conditions on the equation of state (B.1) give $p^{\mathrm{HRM}}\left(\tau, s_{R}, Y_{R}\right)-p^{\mathrm{HRM}}\left(\tau_{R}^{*}, s_{R}, Y_{R}\right)<0$ (resp. $p^{\mathrm{HRM}}\left(\tau, s_{R}, Y_{R}\right)-$ $\left.p^{\mathrm{HRM}}\left(\tau_{R}^{*}, s_{R}, Y_{R}\right)>0\right)$ and together with hypothesis $\mathrm{B.3}$ this yields $\phi^{\prime}(\tau) \geq 0$ (resp. $\phi^{\prime}(\tau) \leq 0$ ). As $\phi\left(\tau_{R}^{*}\right)=0$, we obtain that $\phi\left(\tau_{R}\right)>\phi\left(\tau_{R}^{*}\right)=0$ for $\tau \in$ $I\left(\tau_{R}, \tau_{R}^{*}\right)$. Using the Riemann invariant jump relation $\left(e_{R}^{*}-\frac{\Pi^{*}}{2 a^{2}}\right)=\left(e_{R}-\frac{\Pi_{R}}{2 a^{2}}\right)$, one obtains $0<\phi\left(\tau_{R}\right)=e_{R}^{*}-e^{\mathrm{HRM}}\left(\tau_{R}^{*}, s_{R}, Y_{R}\right)-\frac{1}{2 a^{2}}\left(p^{\mathrm{HRM}}\left(\tau_{R}^{*}, s_{R}, Y_{R}\right)-\Pi^{*}\right)^{2}$. The same lines apply for the case $k=L$.

Lemma 2. Let $\theta \in \mathbb{R}$ and $e_{k}^{*, \theta}=E_{k}^{*, \theta}-\left(u_{k}^{*, \theta}\right)^{2} / 2-\left(v_{k}^{*}\right)^{2} / 2$ for $k=L, R$, then we have

$e_{k}^{*, \theta}-e^{H R M}\left(\tau_{k}, s_{k}, Y_{k}\right)-\frac{1}{2 a^{2}}\left(p^{H R M}\left(\tau_{k}, s_{k}, Y_{k}\right)-\Pi^{*}\right)^{2}+\frac{(1-\theta)^{2}\left(u_{R}-u_{L}\right)^{2}}{8} \geq 0, \quad k=L, R$.

Proof. One has $u_{R}^{*, \theta}=u^{*}+(1-\theta)\left(u_{R}-u_{L}\right) / 2, v_{R}^{*}=v_{R}$ and $\Pi^{*, \theta}=\Pi^{*}+(1-$ $\theta) a\left(u_{R}-u_{L}\right) / 2$, and together with A.6) one obtains $e_{R}^{*, \theta}=e_{R}^{*}-(1-\theta)^{2}\left(u_{R}-\right.$ $\left.u_{L}\right)^{2} / 8$. Injecting this relation into $(\overline{B .4}$ provides the desired result.

It is now clear that the inequalities

$$
-\frac{1}{2 a^{2}}\left(p^{\mathrm{HRM}}\left(\tau_{k}^{*}, s_{k}, Y_{k}\right)-\Pi^{*}\right)^{2}+\frac{(1-\theta)^{2}\left(u_{R}-u_{L}\right)^{2}}{8} \leq 0, \quad k=L, R
$$

can help us providing the modified numerical scheme with a discrete entropy inequality.

Proposition 2. Let $s_{k}^{*, \theta}=s^{H R M}\left(\tau_{k}^{*, \theta}, e_{k}^{*, \theta}, Y_{k}^{*, \theta}\right)$ for $k=L, R$. If assumption (B.6) is verified, we have

$$
s_{k}^{*, \theta} \geq s_{k}
$$


Inequality B.7 implies that the modified scheme A.10 for the acoustic step (which may also be rewritten (7)-(8)) verifies the following discrete entropy inequality

$$
s^{H R M}\left(\tau_{j}^{n+1-}, e_{j}^{n+1-}, Y_{j}^{n+1-}\right) \geq s^{H R M}\left(\tau_{j}^{n}, e_{j}^{n}, Y_{j}^{n}\right) .
$$

Proof. Let $k=L, R$, under hypothesis (B.6), we have $e_{k}^{*, \theta} \geq e^{\operatorname{HRM}}\left(\tau_{k}^{*}, s_{k}, Y_{k}\right)$. Besides, we have $Y_{k}^{*, \theta}=Y_{k}, \tau_{k}^{*, \theta}=\tau_{k}^{*}$ so that $e^{\operatorname{HRM}}\left(\tau_{k}^{*}, s_{k}, Y_{k}\right)=e^{\operatorname{HRM}}\left(\tau_{k}^{*, \theta}, s_{k}, Y_{k}^{*, \theta}\right)$. Assumptions (B.1) on the equation of state imply that $\epsilon \mapsto s^{\operatorname{HRM}}\left(\tau_{k}^{*, \theta}, \epsilon, Y_{k}^{*, \theta}\right)$ is increasing, thus

$$
s^{\operatorname{HRM}}\left(\tau_{k}^{*, \theta}, e_{k}^{*, \theta}, Y_{k}^{*, \theta}\right) \geq s^{\operatorname{HRM}}\left(\tau_{k}^{*, \theta}, e^{\operatorname{HRM}}\left(\tau_{k}^{*, \theta}, s_{k}, Y_{k}^{*, \theta}\right), Y_{k}^{*, \theta}\right)=s_{k}
$$

and inequality $\mathrm{B} .7$ is verified.

Under CFL stability condition (9), the numerical scheme (A.10) for the acoustic step is a convex combination. Furthermore, functions $u \mapsto-\frac{u^{2}}{2}$ and $v \mapsto-\frac{v^{2}}{2}$ are concave, so that

$$
e_{i}^{n+1-} \geq \tilde{e}_{i}^{n+1-}
$$

where $e_{i j}^{n, *, \theta}=E_{i j}^{n, *, \theta}-\left(u_{i j}^{n, *, \theta}\right)^{2} / 2-\left(v_{i j}^{n, *, \theta}\right)^{2} / 2$ and

$$
\tilde{e}_{i}^{n+1-}=\left(1-\tau_{i}^{n} \Delta t \sum_{j \in N(i)} \sigma_{i j} a_{i j}\right) e_{i}^{n}+\tau_{i}^{n} \Delta t \sum_{j \in N(i)} \sigma_{i j} a_{i j} e_{i j}^{n, *, \theta} .
$$

As $\epsilon \mapsto s^{\mathrm{HRM}}\left(\tau_{j}^{n+1-}, \epsilon, Y_{j}^{n+1-}\right)$ is increasing, we obtain

$$
s^{\mathrm{HRM}}\left(\tau_{j}^{n+1-}, e_{j}^{n+1-}, Y_{j}^{n+1-}\right) \geq s^{\mathrm{HRM}}\left(\tau_{j}^{n+1-}, \tilde{e}_{j}^{n+1-}, Y_{j}^{n+1-}\right) .
$$

By definition of $\tilde{e}_{j}^{n+1-}$ and using convex combinations A.10 for variables $\tau$ and $Y$, together with the concave property of function $(\tau, s, Y) \mapsto s^{\mathrm{HRM}}$ yield $s^{\mathrm{HRM}}\left(\tau_{j}^{n+1-}, \tilde{e}_{j}^{n+1-}, Y_{j}^{n+1-}\right) \geq\left(1-\tau_{i}^{n} \Delta t \sum_{j \in N(i)} \sigma_{i j} a_{i j}\right) s_{i}^{n}+\tau_{i}^{n} \Delta t \sum_{j \in N(i)} \sigma_{i j} a_{i j} s_{i j}^{n, *, \theta}$, where $s_{i j}^{n, *, \theta}=s^{\operatorname{HRM}}\left(\tau_{i j}^{n, *, \theta}, e_{i j}^{n, *, \theta}, Y_{i j}^{n, *, \theta}\right)$. We inject inequality B.7 to obtain

$$
s^{\mathrm{HRM}}\left(\tau_{j}^{n+1-}, \tilde{e}_{j}^{n+1-}, Y_{j}^{n+1-}\right) \geq s^{\mathrm{HRM}}\left(\tau_{j}^{n}, e_{j}^{n}, Y_{j}^{n}\right) .
$$

Finally, we use (B.9) and (B.10) to obtain the desired discrete entropy inequality B.8. 
We can now state the following entropy property for the LPS-EX $(\theta)$ scheme.

Proposition 3. If the assumptions (B.6), (9) and (13) are verified, then the $L P S-E X(\theta)$ scheme defined by (7)-(11)-(14) verifies the following discrete entropy inequality

$$
\begin{gathered}
\rho_{i}^{n+1} s^{H R M}\left(\tau_{i}^{n+1}, e_{i}^{n+1}, Y_{i}^{n+1}\right) \geq \rho_{i}^{n} s^{H R M}\left(\tau_{i}^{n}, e_{i}^{n}, Y_{i}^{n}\right)-\Delta t \sum_{j \in N(i)} \sigma_{i j}\left(u_{i j}^{*}\right)^{+} \rho_{i}^{n+1-} s^{H R M}\left(\tau_{i}^{n+1-}, e_{i}^{n+1-}, Y_{i}^{n+1-}\right) \\
-\Delta t \sum_{j \in N(i)} \sigma_{i j}\left(u_{i j}^{*}\right)^{-} \rho_{j}^{n+1-} s^{H R M}\left(\tau_{j}^{n+1-}, e_{j}^{n+1-}, Y_{j}^{n+1-}\right) . \quad \text { (B.11) }
\end{gathered}
$$

Proof. Thanks to proposition 2, we have for the acoustic step (7) the following inequality

$$
s^{\mathrm{HRM}}\left(\tau_{j}^{n+1-}, e_{j}^{n+1-}, Y_{j}^{n+1-}\right) \geq s^{\mathrm{HRM}}\left(\tau_{j}^{n}, e_{j}^{n}, Y_{j}^{n}\right) .
$$

The projection step (11) may be rewritten for $\varphi \in\{\rho Y, \rho, \rho \mathbf{u}, \rho E\}$ under the form

$$
\bar{\varphi}_{i}=\left(1+\Delta t \sum_{j \in N(i)} \sigma_{i j}\left(u_{i j}^{*}\right)^{-}\right) \varphi_{i}^{n+1-}-\Delta t \sum_{j \in N(i)} \sigma_{i j}\left(u_{i j}^{*}\right)^{-} \varphi_{j}^{n+1-},
$$

which is a convex combination under the CFL stability condition (13). As function $(\rho Y, \rho, \rho u, \rho v, \rho E) \mapsto\left(\rho s^{\mathrm{HRM}}\right)(\tau, e, Y)$ is concave, we obtain

$$
\begin{gathered}
\bar{\rho}_{i} s^{\mathrm{HRM}}\left(\bar{\tau}_{i}, \bar{e}_{i}, \bar{Y}_{i}\right) \geq\left(1+\Delta t \sum_{j \in N(i)} \sigma_{i j}\left(u_{i j}^{*}\right)^{-}\right) \rho_{i}^{n+1-} s^{\mathrm{HRM}}\left(\tau_{i}^{n+1-}, e_{i}^{n+1-}, Y_{i}^{n+1-}\right) \\
-\Delta t \sum_{j \in N(i)} \sigma_{i j}\left(u_{i j}^{*}\right)^{-} \rho_{j}^{n+1-} s^{\mathrm{HRM}}\left(\tau_{j}^{n+1-}, e_{j}^{n+1-}, Y_{j}^{n+1-}\right) .
\end{gathered}
$$

For the phase transition step (14), we have the following convex combination

$$
Y_{i}^{n+1}=\frac{1}{1+\lambda_{0} \Delta t} \bar{Y}_{i}+\frac{\lambda_{0} \Delta t}{1+\lambda_{0} \Delta t} Y^{*}\left(\bar{\rho}_{i}, \bar{e}_{i}\right) .
$$

As $(\tau, e, Y) \mapsto s^{\operatorname{HRM}}(\tau, e, Y)$ is concave, we obtain

$$
s^{\mathrm{HRM}}\left(\tau_{i}^{n+1}, e_{i}^{n+1}, Y_{i}^{n+1}\right) \geq \frac{1}{1+\lambda_{0} \Delta t} s^{\mathrm{HRM}}\left(\tau_{i}^{n+1}, e_{i}^{n+1}, \bar{Y}_{i}\right)+\frac{\lambda_{0} \Delta t}{1+\lambda_{0} \Delta t} s^{\mathrm{HRM}}\left(\tau_{i}^{n+1}, e_{i}^{n+1}, Y^{*}\left(\bar{\rho}_{i}, \bar{e}_{i}\right)\right) .
$$

Using $\tau_{i}^{n+1}=\bar{\tau}_{i}, e_{i}^{n+1}=\bar{e}_{i}$ together with the fact that function $Y \mapsto s^{\operatorname{HRM}}(\tau, e, Y)$ 
is minimal at $Y=Y^{*}(\rho, e)$ yield

$$
\rho_{i}^{n+1} s^{\mathrm{HRM}}\left(\tau_{i}^{n+1}, e_{i}^{n+1}, Y_{i}^{n+1}\right) \geq \bar{\rho}_{i} s^{\mathrm{HRM}}\left(\bar{\tau}_{i}, \bar{e}_{i}, \bar{Y}_{i}\right)
$$

We combine (B.12), (B.13), (B.14) and the acoustic scheme for $\tau(7)$ to obtain the discrete entropy inequality (B.11) for the overall numerical scheme LPS$\operatorname{EX}(\theta)$.

We proved in proposition 3 a discrete entropy inequality for $\operatorname{LPS}-\operatorname{EX}(\theta)$ scheme under the condition $(\bar{B} .6)$ on modification $\theta$. Studying $L^{2}$ stability properties of numerical schemes LPS-EX $(\theta)$ and $\operatorname{LPS}-\operatorname{IMEX}(\theta)$ for all value of $\theta \geq 0$ is still an open problem. 


\section{References}

[1] D. McWilliam, R. Duggins, Speed of sound in bubbly liquids, Proc Instn Mech Engrs 1969184 (3C) (1969) pp. 102-107.

[2] S. Kieffer, Sound speed in liquid-gas mixtures: Water-air and water-steam, Journal of Geophysical Research 82 (20) (1977) pp. 2895-2904.

[3] Z. Bilicki, J. Kestin, Physical aspects of the relaxation model in two-phase flow, Proceedings of the Royal Society of London A: Mathematical, Physical and Engineering Sciences 428 (1875) (1990) pp. 379-397.

[4] P. Downar-Zapolski, Z. Bilicki, L. Bolle, J. Franco, The non-equilibrium relaxation model for one-dimensional flashing liquid flow., Int. J. Multiphase Flow 22 (3) (1996) pp. 473-483.

[5] S. Clerc, Numerical simulation of the homogeneous equilibrium model for two-phase flows, Journal of Computational Physics 161 (1) (2000) pp. 354375.

[6] A. Ambroso, C. Chalons, F. Coquel, E. Godlewski, F. Lagoutière, P.-A. Raviart, The coupling of homogeneous models for two-phase flows, Int. Journal for Finite Volume 4 (1) (2007) pp. 39-54.

[7] O. Hurisse, Application of an homogeneous model to simulate the heating of two-phase flows, Int. J. on Finite Vol.

[8] E. Turkel, Preconditioned methods for solving the incompressible and low speed compressible equations, Journal of Computational Physics 72 (2) (1987) pp. 277-298.

[9] H. Guillard, C. Viozat, On the behaviour of upwind schemes in the low mach number limit, Computers \& Fluids 28 (1) (1999) pp. 63-86.

[10] P. Degond, S. Jin, J.-G. Liu, Mach-number uniform asymptotic-preserving gauge schemes for compressible flows., Bull. Inst. Math., Acad. Sin. (N.S.) 2 (4) (2007) pp. 851-892.

[11] B. Braconnier, B. Nkonga, An all-speed relaxation scheme for interface flows with surface tension., J. Comput. Phys. 228 (16) (2009) pp. 57225739 . 
[12] S. Dellacherie, Analysis of Godunov type schemes applied to the compressible Euler system at low Mach number., J. Comput. Phys. 229 (4) (2010) pp. 978-1016.

[13] M. Tang, Second order all speed method for the isentropic euler equations, Kinet. Relat. Models 5 (1) (2012) pp. 155-184.

[14] F. Cordier, P. Degond, A. Kumbaro, An asymptotic-preserving all-speed scheme for the Euler and Navier-Stokes equations., J. Comput. Phys. 231 (17) (2012) pp. 5685-5704.

[15] J. Haack, S. Jin, J.-G. Liu, An all-speed asymptotic-preserving method for the isentropic euler and navier-stokes equations, Commun. Comput. Phys (2012) pp. 955-980.

[16] J.-P.Braeunig, D. Chauveheid, J.-M. Ghidaglia, A totally eulerian finite volume solver for multi-material fluid flows iii: The low mach number case, European Journal of Mechanics - B/Fluids 42 (2013) pp. 10-19.

[17] S. Dellacherie, J. Jung, P. Omnes, P.-A. Raviart, Construction of modified Godunov-type schemes accurate at any Mach number for the compressible Euler system., Math. Models Methods Appl. Sci. 26 (13) (2016) pp. 25252615 .

[18] C. Chalons, M. Girardin, S. Kokh, An all-regime lagrange-projection like scheme for the gas dynamics equations on unstructured meshes, Communications in Computational Physics 20 (1) (2016) pp. 188-233.

[19] M. Bernard, S. Dellacherie, G. Faccanoni, B. Grec, Y. Penel, Study of a low Mach nuclear core model for two-phase flows with phase transition I: Stiffened gas law., ESAIM, Math. Model. Numer. Anal. 48 (6) (2014) pp. 1639-1679.

[20] N. Grenier, J.-P. Vila, P. Villedieu, An accurate low-Mach scheme for a compressible two-fluid model applied to free-surface flows., J. Comput. Phys. 252 (2013) pp. 1-19.

[21] S. LeMartelot, B. Nkonga, R. Saurel, Liquid and liquid-gas flows at all speeds., J. Comput. Phys. 255 (2013) pp. 53-82. 
[22] F. Coquel, Q. L. Nguyen, M. Postel, Q. H. Tran, Entropy-satisfying relaxation method with large time-steps for euler ibvps, Math. Comput. 79 (271) (2010) pp. 1493-1533.

[23] C. Chalons, M. Girardin, S. Kokh, Large time step and asymptotic preserving numerical schemes for the gas dynamics equations with source terms., SIAM J. Sci. Comput. 35 (6) (2013) pp. a2874-a2902.

[24] M. Baer, J. Nunziato, A two-phase mixture theory for the deflagration-todetonation transition (DDT) in reactive granular materials., Int. J. Multiphase Flow 12 (1986) pp. 861-889.

[25] L. Cheng, D. Drew, R. Lahey, An analysis of wave propagation in bubbly two-component, two-phase flow., Journal of Heat Transfer 107 (1985) pp. $402-408$.

[26] B. Després, E. Labourasse, F. Lagoutière, I. Marmajou, An antidissipative transport scheme on unstructured meshes for multicomponent flows, Int. J. Finite. Vol. Meth. (2010) pp. 30-65.

[27] T. Gasc, F. De Vuyst, Suitable formulations of Lagrange remap finite volume schemes for manycore/GPU architectures., in: Finite volumes for complex applications VII - elliptic, parabolic and hyperbolic problems. Proceedings of the FVCA 7, Berlin, Germany, June 15-20, 2014. Vol. II, Cham: Springer, 2014, pp. pp. 607-616.

[28] I. Suliciu, On the thermodynamics of rate-type fluids and phase transitions. i. rate-type fluids, International Journal of Engineering Science 36 (9) (1998) pp. 921-947.

[29] F. Bouchut, Nonlinear stability of finite volume methods for hyperbolic conservation laws and well-balanced schemes for sources, Basel: Birkhäuser, 2004 .

[30] C.Chalons, J.-F. Coulombel, Relaxation approximation of the euler equations, Journal of Mathematical Analysis and Applications 348 (2) (2008) pp. $872-893$.

[31] C.Chalons, F. Coquel, S. Kokh, N. Spillane, Large Time-Step Numerical Scheme for the Seven-Equation Model of Compressible Two-Phase Flows, Springer Berlin Heidelberg, Berlin, Heidelberg, 2011, pp. pp. 225-233. 
[32] A. Harten, P. Lax, B. van Leer, On upstream differencing and Godunovtype schemes for hyperbolic conservation laws., SIAM Rev. 25 (1983) pp. 35-61. 